\title{
SYMBOLS, ABBREVIATIONS AND UNITS
}

\author{
WORKING PARTY \\ STANDARDIZATION OF LUNG FUNCTION TESTS \\ EUROPEAN COMMUNITY FOR STEEL AND COAL
}

Ph. H. Quanjer, G.J. Tammeling, J.E. Cotes, L.M. Fabbri, H. Matthys, O.F. Pedersen, R. Peslin, J. Roca, P.J. Sterk, W.T. Ulmer, J.-C. Yernault

\section{INTRODUCTION}

The recommendations in this 1993 update do not differ materially from those in the previous report of the EUROPEAN COMMUNTTY FOR STEFL AND COAL [1]. However, the list of abbreviations has been extended with respect to the items covered; in addition the European Community has expanded since the previous report, and the report has accordingly been updated to cover the languages spoken in each of the member states.

\section{SYMBOLS}

\subsection{Symbols for quantities}

Symbols are used to designate specific quantities, including basic quantities (e.g. volume, time, pressure, amount of chemical substance) and derived quantities (e.g. volume by unit time). Letters from the Latin or Greek alphabet are commonly employed as symbols, either roman type as in the USA or italics as recommended by the EURopean Society for Clinical Respiratory Physiology [2] and adopted in the 1983 report of the ECSC. As the number of letters available is limited, inevitably one symbol may be used to designate more than one quantity (e.g. concentration of chemical substance and compliance). Symbols for quantities may be specified by one or more subscripts and/or prescripts (abbreviations) and/or modifying signs (dashes, dots, primes), e.g. $\Delta V_{50^{\circ}}$. Subscripts other than numbers are printed in roman small capitals or lower case letters. The order of specification is location (where), time (when), condition or quality (what, how). Specifications are printed either in line with the primary symbol or as subscripts. When more than one subscript is used, these are separated by a comma.

The working party is very grateful to Prof. N. Siafakas, Drs. B. Bouros, N. Tzanakis and F. Vlaserou for the translation of the tables into Greek, and to Prof. J.H. Paiva de Carvalho for the translation into Portuguese.

\subsection{Symbols for units}

Symbols for SI and non-SI units are roman lower case letters, unless the name of the unit is derived from a proper name, in which case it consists of a capital roman letter (except ohm, $\Omega$ ), or a capital roman and a lower case letter. Prefixes are used to modify symbols for units and are single roman capitals or lower case letters (except deca, da). In respiratory physiology and medicine, the SI base units are extended with SI derived units and SI prefixes. Non-SI units which are widely applied in everyday life are retained for general use with the SI.

\section{ABBREVIATIONS}

Abbreviations are employed to facilitate written and spoken communication and are commonly specific to individual languages. However, there is a tendency for many abbreviations to be adopted in the various languages spoken within the European Community; they are also used in mathematical formulae and equations. Such abbreviations have acquired the attributes of symbols. Commonly accepted standard abbreviations for quantities are usually written in one or more capital letters, but there are many exceptions (e.g. Hb, cAMP, co-A, ATP-ase). The abbreviations can be specified by one or more subscripts and/or modifying signs (dashes, dots, primes). Subscripts are numbers or letters printed in roman small capital or small lower case type, e.g. $\mathrm{FEV}_{1}$.

\section{UNITS}

\subsection{SI base units}

\begin{tabular}{lll}
\hline Name of quantity & $\begin{array}{l}\text { Name of } \\
\text { unit }\end{array}$ & $\begin{array}{l}\text { Symbol for } \\
\text { unit }\end{array}$ \\
\hline length & metre & $\mathrm{m}$ \\
mass & kilogramme & $\mathrm{kg}$ \\
time & second & $\mathrm{s}$ \\
electric current & ampere & $\mathrm{A}$ \\
thermodynamic temperature & kelvin & $\mathrm{K}$ amount \\
of substance & mole & mol \\
luminous intensity & candela & $\mathrm{cd}$ \\
\hline
\end{tabular}




\subsection{Selected SI prefixes}

\begin{tabular}{crc}
\hline Factor & Prefix & Symbol \\
\hline $10^{6}$ & mega & $\mathrm{M}$ \\
$10^{3}$ & kilo & $\mathrm{k}$ \\
$10^{2}$ & hecto & $\mathrm{h}$ \\
$10^{1}$ & deca & da \\
$10^{-1}$ & deci & $\mathrm{d}$ \\
$10^{-2}$ & centi & $\mathrm{c}$ \\
$10^{-3}$ & milli & $\mathrm{m}$ \\
$10^{-6}$ & micro & $\mu$ \\
$10^{-9}$ & nano & $\mathrm{n}$ \\
\hline
\end{tabular}

\subsection{Selected SI derived units}

\begin{tabular}{|c|c|c|c|}
\hline Name of quantity & $\begin{array}{c}\text { Name of } \\
\text { unit }\end{array}$ & $\begin{array}{l}\text { Symbol } \\
\text { for unit }\end{array}$ & $\begin{array}{l}\text { Definition } \\
\text { of unit }\end{array}$ \\
\hline frequency & hertz & $\mathrm{Hz}$ & $\mathrm{s}^{-1}$ \\
\hline force & newton & $\mathrm{N}$ & $\mathrm{m} \cdot \mathrm{kg} \cdot \mathrm{s}^{-2}$ \\
\hline pressure & pascal & $\mathrm{Pa}$ & $\mathrm{N} \cdot \mathrm{m}^{-2}$ \\
\hline energy, work & joule & $\mathbf{J}$ & $\mathrm{N} \cdot \mathrm{m}$ \\
\hline power & watt & W & $\mathrm{J} \cdot \mathrm{s}^{-1}$ \\
\hline Celsius temperature & $\begin{array}{l}\text { degree } \\
\text { Celsius }\end{array}$ & ${ }^{\circ} \mathrm{C}$ & * \\
\hline mass density & & & $\mathrm{kg} \cdot \mathrm{m}^{-3}$ \\
\hline dynamic viscosity & poise & $\mathbf{P}$ & $10^{-1} \cdot \mathrm{Pa} \cdot \mathrm{s}$ \\
\hline kinematic viscosity & stokes & St & $10^{-4} \cdot \mathrm{m}^{2} \cdot \mathrm{s}^{-1}$ \\
\hline
\end{tabular}

\subsection{Selected non-SI units retained}

\begin{tabular}{lrrr}
\hline Name of quantity & $\begin{array}{r}\text { Name of } \\
\text { unit }\end{array}$ & $\begin{array}{r}\text { Symbol } \\
\text { for unit }\end{array}$ & $\begin{array}{r}\text { Definition } \\
\text { of unit }\end{array}$ \\
\hline time & $\begin{array}{r}\text { minute } \\
\text { hour }\end{array}$ & $\begin{array}{r}\text { min } \\
\text { day }\end{array}$ & $60 \mathrm{~s}$ \\
& year & $\mathrm{d}$ & $8600 \mathrm{~s}$ \\
& litre & $\mathrm{a}$ & $36400 \mathrm{~s}$ \\
volume & $l$ & $10^{-3} \cdot \mathrm{m}^{3}$ \\
blood pressure & millimetre & $\mathrm{mmHg}$ & $133.322 \mathrm{~Pa}$ \\
& of mercury & & \\
\hline
\end{tabular}

\section{Comments}

1 The American and European recommendations differ with respect to pressure, amount of substance (gas) and time.

2 The working party recommends that time be reported in second for quantities which relate to instantaneous events; time averaged quantities should be reported in units which are appropriate to the length of time over which they are obtained (e.g. seconds, minutes, hours). For special purposes the units day (d), month (m) and year (a) may be used.

3 Volume of gas is usually expressed in $l_{\text {BTPS }}$. Note that in this case the subscript refers to the unit. Unlike a previous recommendation the symbol for litre is now printed in italics, as the distinction between 1 (one) and 1 (for litre) with most fonts is commonly too subtle.

4 Amount of gas: mmol.
5 Partial pressure of gas: $\mathrm{kPa}$.

6 Blood pressure: $\mathrm{mmHg}$ (temporarily permitted); $\mathrm{kPa}$.

\subsection{Special notations and mathematical operations}

$\overline{\mathrm{X}} \quad$ mean value of $\mathrm{X}$

$\mathrm{X}^{\prime} \quad$ time derivative of $\mathrm{X}$

$X^{\prime \prime} \quad$ second time derivative of $X$

$\overline{\mathrm{X}}^{\prime}$ time averaged value of $\overline{\mathrm{X}}^{\prime}$ (to be specified)

$\Delta \mathrm{X}_{\mathrm{A}} \quad$ change of $\mathrm{X}$ for specification $\mathrm{A}$

$\mathrm{X}_{\mathrm{A}-\mathrm{B}}$ difference between $\mathrm{X}$-values for specifications $\mathrm{A}$ and $\mathrm{B}$

$X_{A, B}$ different specifications of $X$ are separated by a comma

$\% \mathrm{X} \quad \mathrm{X}$ as a percentage of the reference value

$\mathrm{X} \% \mathrm{Y} \quad \mathrm{X}$ as a percentage of $\mathrm{Y}$

$\mathrm{X} / \mathrm{Y}$ division is indicated by a solidus (stroke)

$\mathrm{X} \cdot \mathrm{Y}$ multiplication is indicated by a raised dot

$\mathrm{X} \cdot \mathrm{Y} \cdot \mathrm{Z}^{-1}$

$\mathrm{X} \cdot \mathrm{Y} / \mathrm{Z}$

$(X \cdot Y)^{-1}$

\} examples of mathematical notations

\section{Comments}

1 In most American recommendations, the notation $\dot{X}$ is used both for the instantaneous and the timeaveraged value of the first time derivative of $\mathrm{X}$, or the lower case form is used as well (e.g. x). In accordance with the SEPCR recommendation [2] the working party recommends $\mathrm{X}^{\prime}$ for the instantaneous time derivative and $\overline{\mathrm{X}}$ ' for the averaged time derivative.

2 The second time derivative of $\mathrm{X}$ is usually indicated by $\ddot{X}$ in the USA; the present recommendation is $\mathrm{X}^{\prime \prime}$.

3 The notation $\mathrm{X} / \mathrm{Y}$ is allowed, except with complex notations. More than one solidus should never be used in a notation.

\subsection{Abbreviations, symbols and units for lung} function indices

The following tables contain abbreviation, symbols and units for some commonly used lung function indices. Gas volumes are at BTPS, unless indicated otherwise. Qualifying abbreviations are usually one or more roman lower case letters. However, for several qualifications, capital letters are (also) accepted.

\section{References}

1. Quanjer $\mathrm{PhH}$ (ed.). - Standardized lung function testing. Bull Europ Physiopathol Respir 1983; 19 (suppl 5): 195.

2. Matthys $\mathrm{H}$ (ed.). - Clinical respiratory physiology: Abbreviations, symbols and units, definitions. Working document of the SEPCR. Literae Medicinales Thomae, 1978. 
ption - Quantity - Unit

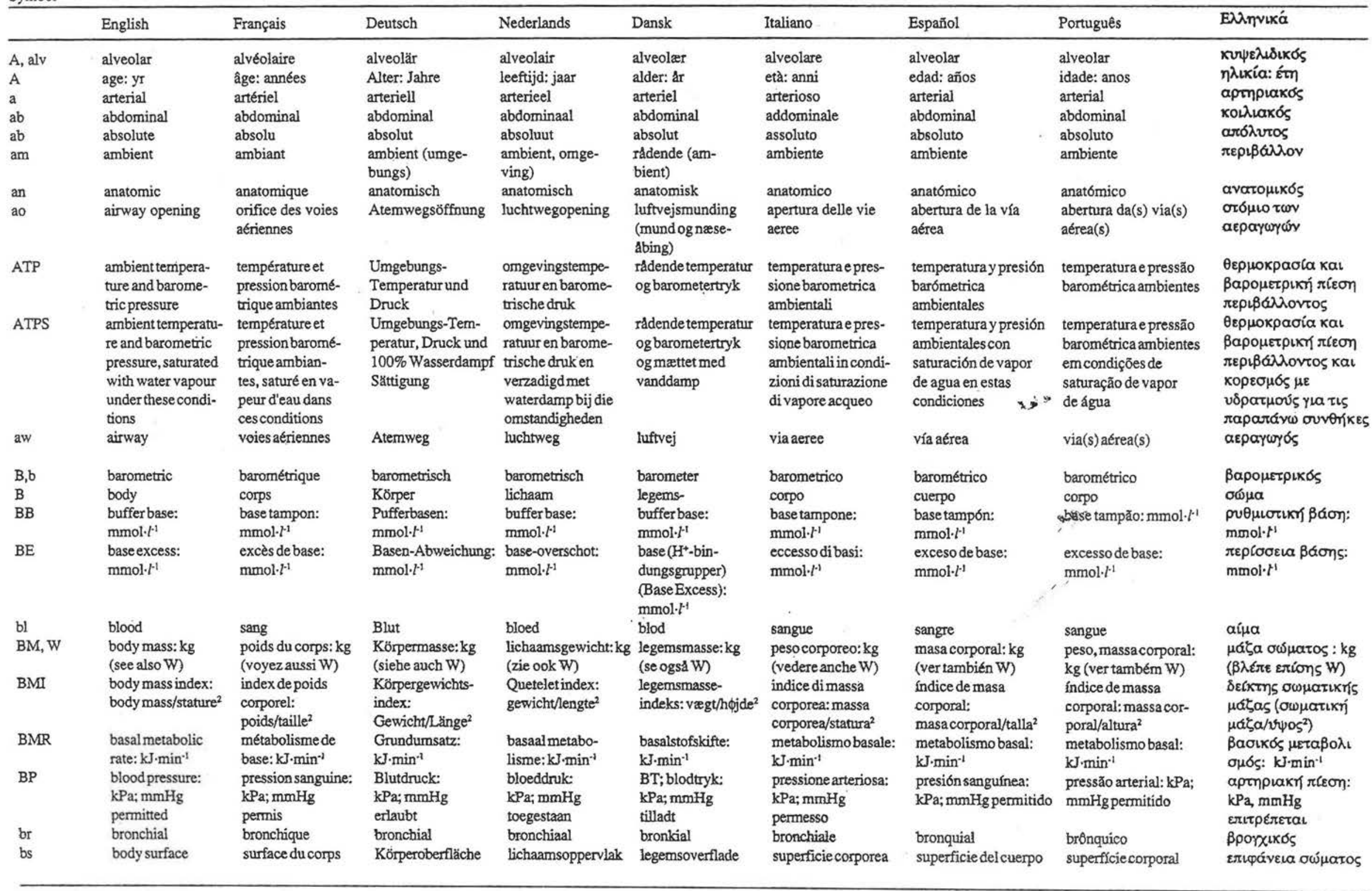




\begin{tabular}{|c|c|c|c|c|c|c|c|c|c|}
\hline & English & Français & Deutsch & Nederlands & Dansk & Italiano & Español & Português & 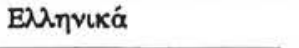 \\
\hline BSA & $\begin{array}{l}\text { body surface area: } \\
\mathrm{m}^{2}\end{array}$ & $\begin{array}{l}\text { surface corporelle: } \\
\mathrm{m}^{2}\end{array}$ & $\begin{array}{l}\text { Körperoberfläche: } \\
\mathrm{m}^{2}\end{array}$ & $\begin{array}{l}\text { lichaamsoppervlak: } \\
\mathrm{m}^{2}\end{array}$ & $\begin{array}{l}\text { legemsoverflade: } \\
\mathrm{m}^{2}\end{array}$ & $\begin{array}{l}\text { area della superficie } \\
\text { corporea: } \mathrm{m}^{2}\end{array}$ & aérea corporal: $\mathrm{m}^{2}$ & $\begin{array}{l}\text { área de superfície } \\
\text { corporal: } \mathrm{m}^{2}\end{array}$ & 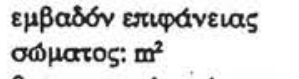 \\
\hline BTPS & $\begin{array}{l}\text { body temperature, } \\
\text { barometric } \\
\text { pressure and satu- } \\
\text { rated with water } \\
\text { vapour under these } \\
\text { conditions }\end{array}$ & $\begin{array}{l}\text { température du } \\
\text { corps, pression } \\
\text { barométrique, } \\
\text { saturé en vapeur } \\
\text { d'eau dans ces } \\
\text { conditions }\end{array}$ & $\begin{array}{l}\text { Körper-Temperatur, } \\
\text { Druck und 100\% } \\
\text { Wasserdampf } \\
\text { Sättigung }\end{array}$ & $\begin{array}{l}\text { lichaamstempera- } \\
\text { tuur en druk, ver- } \\
\text { zadigd met water- } \\
\text { damp bij deze om- } \\
\text { standigheden }\end{array}$ & $\begin{array}{l}\text { legemstemperatur, } \\
\text { rádende barometer- } \\
\text { tryk og mættet } \\
\text { vanddamp }\end{array}$ & $\begin{array}{l}\text { temperatura corporea } \\
\text { pressione barome- } \\
\text { trica ambientale in } \\
\text { condizioni di satura- } \\
\text { zione di vapore } \\
\text { acqueo }\end{array}$ & $\begin{array}{l}\text { temperatura corporal, } \\
\text { presión barométrica } \\
\text { y saturación de vapor } \\
\text { de agua en estas } \\
\text { condiciones }\end{array}$ & $\begin{array}{l}\text { temperatura corporal, } \\
\text { pressão barométrica, } \\
\text { em condiçōes de } \\
\text { saturação de vapor } \\
\text { de água }\end{array}$ & 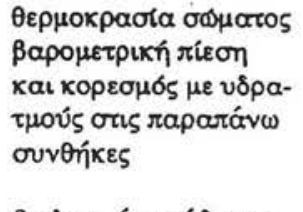 \\
\hline $\mathrm{BU}$ & $\begin{array}{l}\text { biological unit for } \\
\text { allergen extracts } \\
\mathrm{BU} \cdot \mathrm{ml}^{-1}\end{array}$ & $\begin{array}{l}\text { unité biologique } \\
\text { d'extrait d'allergène: } \\
\text { BU } \cdot \mathrm{ml}^{-1}\end{array}$ & $\begin{array}{l}\text { biologische Einheit: } \\
\mathrm{BU} \cdot \mathrm{ml}^{-1}\end{array}$ & $\begin{array}{l}\text { biologische een- } \\
\text { heid voor allergeen- } \\
\text { extracten: } \mathrm{BU} \cdot \mathrm{ml}^{-1}\end{array}$ & $\begin{array}{l}\text { biologisk enhed for } \\
\text { allergenekstrakter: } \\
\text { BU·ml-1 }\end{array}$ & $\begin{array}{l}\text { unità biologiche di } \\
\text { estratti allergenici } \\
\text { BU.ml }\left.\right|^{-1}\end{array}$ & $\begin{array}{l}\text { unidad biológica para } \\
\text { extractos alergénicos: } \\
\text { BU·ml-1 }\end{array}$ & $\begin{array}{l}\text { unidade biologica para } \\
\text { extractos alergénicos: } \\
\text { BU } \cdot \mathrm{ml}^{-1}\end{array}$ & 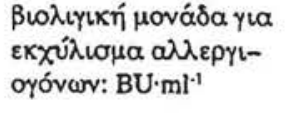 \\
\hline c & cardiac & cardi & kardial & cardiaal & hjerte & cardiaco & cardfaco & cardíaco & карठ \\
\hline c & pulmonary capillary & $\begin{array}{l}\text { capillaire } \\
\text { pulmonaire }\end{array}$ & Lungenkapilaren & pulmonaal capillair & lungekapillær & capillare poimonare & capilar pulmonar & capilar pulmonar & 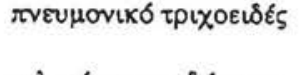 \\
\hline$c^{\prime}$ & $\begin{array}{l}\text { end-capillary } \\
\text { pulmonary }\end{array}$ & $\begin{array}{l}\text { à la fin du } \\
\text { capillaire } \\
\text { pulmonaire }\end{array}$ & $\begin{array}{l}\text { Lungenend- } \\
\text { kapilaren }\end{array}$ & $\begin{array}{l}\text { eind-capillair in de } \\
\text { longcirculatie }\end{array}$ & $\begin{array}{l}\text { ved distale ende af } \\
\text { lungekapillær }\end{array}$ & $\begin{array}{l}\text { alla fine del capillare } \\
\text { polmonare }\end{array}$ & $\begin{array}{l}\text { final del capilar } \\
\text { pulmonar }\end{array}$ & $\begin{array}{l}\text { no extremo do capilar } \\
\text { pulmonar }\end{array}$ & 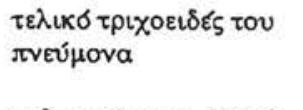 \\
\hline C & compliance: $l \cdot \mathrm{kPa}^{-1}$ & compliance: $l \cdot \mathrm{kPa}^{-1}$ & Compliance: $l \cdot \mathrm{kPa}^{-1}$ & compliantie: $l \cdot \mathrm{kPa}^{-1}$ & $\begin{array}{l}\text { compliance ("efter- } \\
\text { givelighed"): } l \cdot \mathrm{kPa}^{-1}\end{array}$ & compliance: $l \cdot \mathrm{kPa}^{-1}$ & compliancia: $l \cdot \mathrm{kPa} x$ & $\begin{array}{l}\text { "compliance"; distensi- } \\
\text { bilidade: } l \cdot \mathrm{kPa}^{-1}\end{array}$ & $\varepsilon \vee \delta 0 \tau(x o ̛ ́ \eta\rceil \alpha: l \cdot \mathrm{kPa}^{-1}$ \\
\hline$c_{1}$ & $\begin{array}{l}\text { molar concentration } \\
\text { of component i: } \\
\text { mmol. } l^{-1}\end{array}$ & $\begin{array}{l}\text { concentration mol- } \\
\text { aire d'un composant } \\
\text { i: mmol } l^{-1}\end{array}$ & $\begin{array}{l}\text { Molare Konzen- } \\
\text { tration der Kompo- } \\
\text { nente i: mmol } l^{-1}\end{array}$ & $\begin{array}{l}\text { molaire concen- } \\
\text { tratie van stof i: } \\
\text { mmol } l^{-1}\end{array}$ & $\begin{array}{l}\text { koncentration af } \\
\text { komponent i: } \\
\text { mmol } \cdot l^{-1}\end{array}$ & $\begin{array}{l}\text { concentrazione } \\
\text { molare del compo- } \\
\text { nente i: mmol. } l^{-1}\end{array}$ & $\begin{array}{l}\text { concentración molar } \\
\text { del componente i: } \\
\text { mmol } l^{-1}\end{array}$ & $\begin{array}{l}\text { concentração molar do } \\
\text { componente i: } \mathrm{mmol} \cdot l^{-1}\end{array}$ & 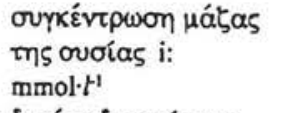 \\
\hline$C_{\mathrm{L}} / V_{\mathrm{L}}\left(\mathrm{s} C_{\mathrm{L}}\right)$ & $\begin{array}{l}\text { volumic (specific) } \\
\text { compliance of the } \\
\text { lung: } \mathrm{kPa}^{-1}\end{array}$ & $\begin{array}{l}\text { compliance spéci- } \\
\text { fique du poumon: } \\
\mathrm{kPa}^{-1}\end{array}$ & $\begin{array}{l}\text { Volumische (spe- } \\
\text { zifische) Com- } \\
\text { pliance: } \mathrm{kPa}^{-1}\end{array}$ & $\begin{array}{l}\text { volumieke (speci- } \\
\text { fieke) compliantie } \\
\text { van de long: } \mathrm{kPa}^{-1}\end{array}$ & $\begin{array}{l}\text { volumetrisk (spe- } \\
\text { cifik) compliance: } \\
\mathrm{kPa}^{-1}\end{array}$ & $\begin{array}{l}\text { compliance } \\
\text { polmonare specifica: } \\
\mathrm{kPa}^{-1}\end{array}$ & $\begin{array}{l}\text { compliancia especifica } \\
\text { del pulmón: } \mathrm{kPa}^{-1}\end{array}$ & $\begin{array}{l}\text { "compliance" especifica } \\
\text { dy pulmão: } \mathrm{kPa}^{-1}\end{array}$ & 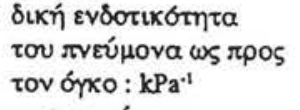 \\
\hline $\mathrm{CC}$ & closing capacity: $l$ & $\begin{array}{l}\text { capacité de ferme- } \\
\text { ture: } l\end{array}$ & $\begin{array}{l}\text { Verschluss } \\
\text { kapazität: } l\end{array}$ & afsluitcapaciteit: $l$ & lukningskapacitet: $l$ & capacità di chiusura: $l$ & capacidad de cierre: $l$ & $\begin{array}{l}\text { capacidade de encerra- } \\
\text { mento: } l\end{array}$ & 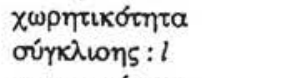 \\
\hline CC\%TLC & $\begin{array}{l}\text { closing capacity as } \\
\text { a percentage of the } \\
\text { total lung capacity }\end{array}$ & $\begin{array}{l}\text { capacité de ferme- } \\
\text { ture en pourcen- } \\
\text { tage de la capacité } \\
\text { pulmonaire totale }\end{array}$ & $\begin{array}{l}\text { Verschluss } \\
\text { kapazität } \\
\text { als Prozent der tota- } \\
\text { len Lungenkapazität }\end{array}$ & $\begin{array}{l}\text { afsluitcapaciteit als } \\
\text { percentage van de } \\
\text { totale longcapaciteit }\end{array}$ & $\begin{array}{l}\mathrm{CC} \text { i procent af } \\
\text { TLC }\end{array}$ & $\begin{array}{l}\text { capacità di chiusura } \\
\text { espressa in percen- } \\
\text { tuale della capacità } \\
\text { polmonare totale } \\
\text { (CC\%CPT) }\end{array}$ & $\begin{array}{l}\text { capacidad de cierre } \\
\text { expresado como } \\
\text { porcentaje de la } \\
\text { capacidad pulmonar } \\
\text { total }\end{array}$ & $\begin{array}{l}\text { capacidade de encerra- } \\
\text { mento em percentagem } \\
\text { de TLC }\end{array}$ & 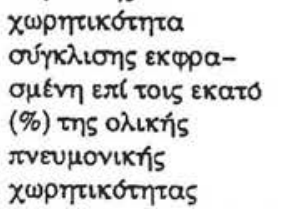 \\
\hline cd & circadian & circadien & Tagesrhythmus & circadisch & $d \phi g n$ & circadiano & circadiano & circadiano & 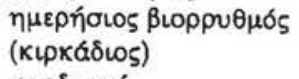 \\
\hline c.o. & $\begin{array}{l}\text { cardiac output: } \\
l \cdot \min ^{-1} \text { (see also } \\
Q^{\prime} \text { ) }\end{array}$ & $\begin{array}{l}\text { débit cardiaque: } \\
\text { l-min } \\
Q^{-1} \text { (voyez aussi }\end{array}$ & $\begin{array}{l}\text { Herzzeitvolumen: } \\
l \cdot \min ^{-1} \text { (siehe auch } \\
\left.Q^{\prime}\right)\end{array}$ & $\begin{array}{l}\text { hartdebiet: } l \cdot \mathrm{min}^{-1} \\
\text { (zie ook } Q^{\prime} \text { ) }\end{array}$ & $\begin{array}{l}\text { hjertets minut- } \\
\text { volumen: } l \cdot \mathrm{min}^{-1} \\
\left.\bar{Q}^{\prime}\right)\end{array}$ & $\begin{array}{l}\text { portata cardiaca: } \\
l \cdot \mathrm{min}^{-1} \text { (vedere anche } \\
\left.\bar{Q}^{\prime}\right)\end{array}$ & $\begin{array}{l}\text { débito cardíaco: } \\
\text { l.min'-1 (ver también } \\
\bar{Q}^{\prime} \text { ) }\end{array}$ & $\begin{array}{l}\text { débito cardíaco: } l \cdot \mathrm{min}^{-1} \\
\text { (ver também } \mathbf{Q}^{\prime} \text { ) }\end{array}$ & 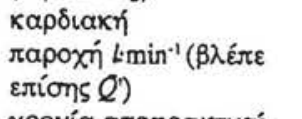 \\
\hline COPD & $\begin{array}{l}\text { chronic obstructive } \\
\text { lung disease }\end{array}$ & $\begin{array}{l}\text { maladie pulmonaire } \\
\text { obstructive } \\
\text { chronique }\end{array}$ & $\begin{array}{l}\text { chronisch obstruk- } \\
\text { tive Lungenkrank- } \\
\text { heit }\end{array}$ & $\begin{array}{l}\text { chronische obstruc- } \\
\text { tieve longziekten }\end{array}$ & $\begin{array}{l}\text { kronisk obstruktiv } \\
\text { lungelidelse }\end{array}$ & $\begin{array}{l}\text { broncopneumopatia } \\
\text { cronica ostruttiva }\end{array}$ & $\begin{array}{l}\text { enfermedad pulmonar } \\
\text { obstructiva crónica }\end{array}$ & $\begin{array}{l}\text { doença pulmonar } \\
\text { obstrutiva crónica }\end{array}$ & 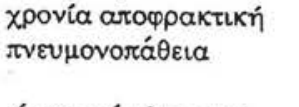 \\
\hline CV & closing volume: I & $\begin{array}{l}\text { volume de fer- } \\
\text { meture: } l\end{array}$ & $\begin{array}{l}\text { Verschluss } \\
\text { volumen: } l\end{array}$ & afsluitvolume: $l$ & $\begin{array}{l}\text { lukningsvolumen: } \\
l\end{array}$ & volume di chiusura: $l$ & volumen de cierre: $l$ & $\begin{array}{l}\text { volume de encerra- } \\
\text { mento: } l\end{array}$ & 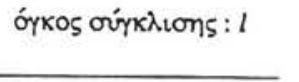 \\
\hline
\end{tabular}




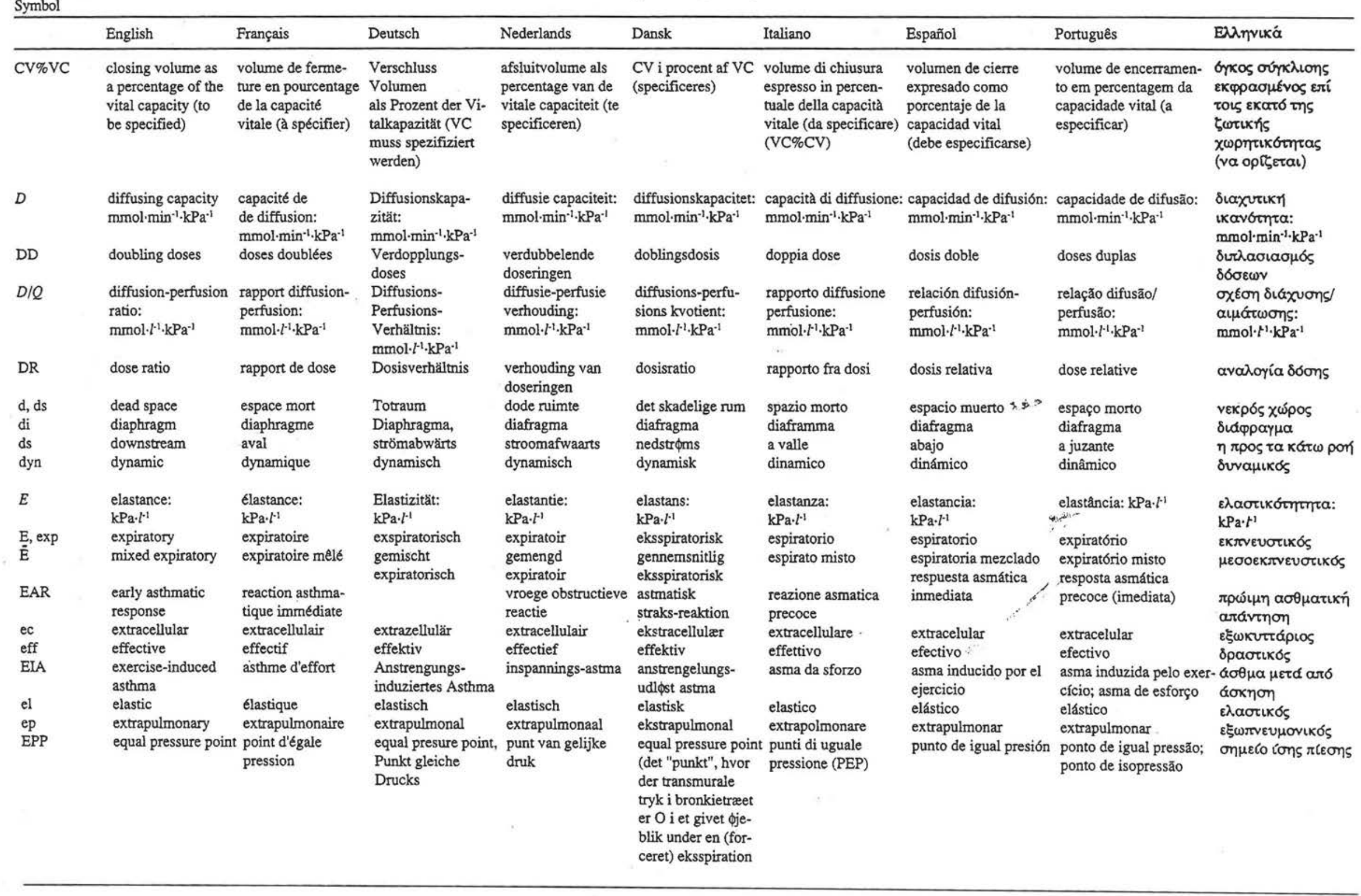




\begin{tabular}{|c|c|c|c|c|c|c|c|c|c|}
\hline & English & Français & Deutsch & Nederlands & Dansk & Italiano & Español & Português & 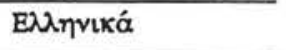 \\
\hline ERV & $\begin{array}{l}\text { expiratory reserve } \\
\text { volume: } l\end{array}$ & $\begin{array}{l}\text { volume de réserve } \\
\text { expiratoire: } l\end{array}$ & $\begin{array}{l}\text { exspiratorisches } \\
\text { Reserve Volu- } \\
\text { men: } l\end{array}$ & $\begin{array}{l}\text { expiratoir reserve } \\
\text { volume: } l\end{array}$ & $\begin{array}{l}\text { eksspiratorisk } \\
\text { reservevolumen: l }\end{array}$ & $\begin{array}{l}\text { volume di riserva } \\
\text { espiratoria: l }\end{array}$ & $\begin{array}{l}\text { volumen de reserva } \\
\text { espiratorio: } l\end{array}$ & $\begin{array}{l}\text { volume de reserva } \\
\text { expiratória: } l\end{array}$ & 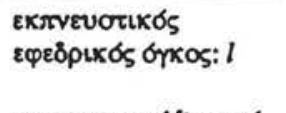 \\
\hline EVC & $\begin{array}{l}\text { expiratory vital } \\
\text { capacity: } l\end{array}$ & $\begin{array}{l}\text { capacite vitale } \\
\text { expiratoire: } l\end{array}$ & $\begin{array}{l}\text { exspiratorische } \\
\text { Vitalkapazităt: } l\end{array}$ & $\begin{array}{l}\text { expiratoire vitale } \\
\text { capaciteit: } l\end{array}$ & $\begin{array}{l}\text { eksspiratorisk VC: } \\
l\end{array}$ & $\begin{array}{l}\text { capacità vitale } \\
\text { espiratoria: } l\end{array}$ & $\begin{array}{l}\text { capacidad vital } \\
\text { espiratoria: } l\end{array}$ & $\begin{array}{l}\text { capacidade vital expira- } \\
\text { tória: } l\end{array}$ & 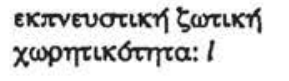 \\
\hline $\mathrm{F}$ & fernale & féminin & weiblich & vrouw & kvindelig & femmina & mujer & feminino & $\gamma u v \alpha\{k \alpha$ \\
\hline f & $\begin{array}{l}\text { functional, } \\
\text { frequency }\end{array}$ & $\begin{array}{l}\text { fonctionnel, } \\
\text { frequence }\end{array}$ & $\begin{array}{l}\text { funktionell, } \\
\text { Frequenz }\end{array}$ & $\begin{array}{l}\text { functioneel, } \\
\text { frequentie }\end{array}$ & $\begin{array}{l}\text { funktionel, } \\
\text { frekvens }\end{array}$ & $\begin{array}{l}\text { funzionale, } \\
\text { frequenza }\end{array}$ & $\begin{array}{l}\text { funcional, } \\
\text { frecuencia }\end{array}$ & $\begin{array}{l}\text { funcional, } \\
\text { frequência }\end{array}$ & 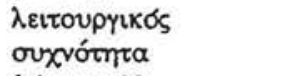 \\
\hline$F$ & force: $N$ & force: $N$ & Kraft: $\mathrm{N}$ & kracht: $\mathrm{N}$ & kraft: N & forza: $\mathrm{N}$ & fuerza: $N$ & força: $N$ & 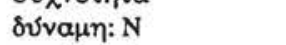 \\
\hline$f_{c}$ & $\begin{array}{l}\text { cardiac frequency: } \\
\min ^{-1}, \mathrm{~s}^{-1}\end{array}$ & $\begin{array}{l}\text { fréquence } \\
\text { cardiaque: } \min ^{-1}, s^{-1}\end{array}$ & $\begin{array}{l}\text { Herzfrequenz: } \\
\mathrm{min}^{-1}, \mathrm{~s}^{-1}\end{array}$ & $\begin{array}{l}\text { hartfrequentie: } \\
\min ^{-1}, \mathrm{~s}^{-1}\end{array}$ & $\begin{array}{l}\text { puls, hjertefrekvens: } \\
\min ^{-1}, \mathrm{~s}^{-1}\end{array}$ & $\begin{array}{l}\text { frequenza cardiaca: } \\
\min ^{-1}, s^{-1}\end{array}$ & $\begin{array}{l}\text { frecuencia cardfaca: } \\
\min ^{-1}, \mathrm{~s}^{-1}\end{array}$ & $\begin{array}{l}\text { frequência cardíaca: } \\
\min ^{-1}, \mathrm{~s}^{-1}\end{array}$ & 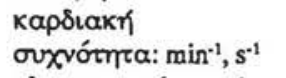 \\
\hline$F_{\mathrm{i}}$ & $\begin{array}{l}\text { fractional concen- } \\
\text { tration of compo- } \\
\text { nent i }\end{array}$ & $\begin{array}{l}\text { concentration frac- } \\
\text { tionnelle d'un } \\
\text { composant } i\end{array}$ & $\begin{array}{l}\text { fraktionelle Kon- } \\
\text { zentration der } \\
\text { Komponente i }\end{array}$ & $\begin{array}{l}\text { fractionele concen- } \\
\text { tratie van substan- } \\
\text { tie } \mathrm{i}\end{array}$ & $\begin{array}{l}\text { koncentration af } \\
\text { komponent } \mathrm{i} \text { : } \\
\text { fraktion }\end{array}$ & $\begin{array}{l}\text { frazione percentuale } \\
\text { del componente } i\end{array}$ & $\begin{array}{l}\text { concentración fraccio- } \\
\text { nal del componente i }\end{array}$ & $\begin{array}{l}\text { concentração percentual } \\
\text { do componente i }\end{array}$ & 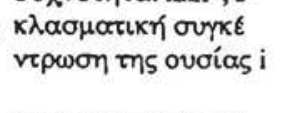 \\
\hline$f_{\mathrm{R}}$ & $\begin{array}{l}\text { breathing frequen- } \\
\text { cy: } \min ^{-1}, s^{-1}\end{array}$ & $\begin{array}{l}\text { fréquence respira- } \\
\text { toire: } \mathrm{min}^{-1}, \mathrm{~s}^{-1}\end{array}$ & $\begin{array}{l}\text { Atemfrequenz: } \\
\min ^{-1}, s^{-1}\end{array}$ & $\begin{array}{l}\text { ademhalingsfre- } \\
\text { quentie: } \min ^{-1}, \mathrm{~s}^{-1}\end{array}$ & $\begin{array}{l}\text { respirationsfre- } \\
\text { kvens: } \min ^{-1}, s^{-1}\end{array}$ & $\begin{array}{l}\text { frequenza respirato- } \\
\text { ria: } \min ^{-1}, s^{-1}\end{array}$ & $\begin{array}{l}\text { frecuencia respiratoria: } \\
\min ^{-1}, s^{-1}\end{array}$ & $\begin{array}{l}\text { frequência respiratória: } \\
\min ^{-1}, s^{-1}\end{array}$ & 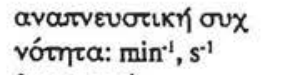 \\
\hline $\mathrm{FEF}_{\text {xकFC }}$ & $\begin{array}{l}\text { forced expiratory } \\
\text { flow when } x \% \text { of } \\
\text { forced expiratory } \\
\text { vital capacity has } \\
\text { been exhaled: } l \cdot \mathrm{s}^{-1}\end{array}$ & $\begin{array}{l}\text { débit expiratoire } \\
\text { forcé lorsque } \mathrm{x} \% \\
\text { de la capacité vi- } \\
\text { tale expiratoire } \\
\text { a été expire: } 1 \cdot \mathrm{s}^{-1}\end{array}$ & $\begin{array}{l}\text { forcierter exspira- } \\
\text { torischer Fluss, } \\
\mathrm{x} \% \text { forcierter } \\
\text { ausgeatmeter } \\
\text { Vitalkapazität : } \\
l \cdot \mathrm{s}^{-1}\end{array}$ & $\begin{array}{l}\text { maximale expira- } \\
\text { ratoire volume- } \\
\text { stroom wanneer } x \% \\
\text { van de geforceerde } \\
\text { vitale capaciteit is } \\
\text { uitgeademd: } l \cdot \mathrm{s}^{-1}\end{array}$ & $\begin{array}{l}\text { forceret eksspirato- } \\
\text { risk volumenstrøm } \\
\text { i det } \text { фjeblik } \\
\mathrm{x} \% \text { af FVC er } \\
\text { eksspireret: } l \cdot \mathrm{s}^{-1}\end{array}$ & $\begin{array}{l}\text { flusso espiratorio } \\
\text { forzato ad un determi- } \\
\text { nato livello percentu- } \\
\text { ale della capacità } \\
\text { vitale forzata: } l \cdot \mathrm{s}^{-1} \\
\left(\mathrm{FEF}_{\mathrm{x} \mathrm{ccvp}}\right)\end{array}$ & $\begin{array}{l}\text { flujo espiratorio forza- } \\
\text { do cuando el } \times \% \text { de la } \\
\text { capacidad vital forzada } \\
\text { ha sido espirado: } l \cdot \mathrm{s}^{*} \cdot \mathrm{s}\end{array}$ & $\begin{array}{l}\text { débito expiratório } \\
\text { forçado quando } x \% \\
\text { da capacidade vital } \\
\text { expiratória forçada } \\
\text { foi expirado: } l \cdot \mathrm{s}^{-1}\end{array}$ & 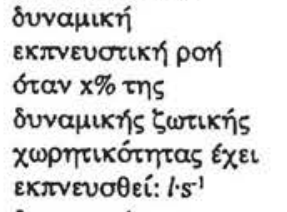 \\
\hline $\mathrm{FEF}_{25-75 \%}$ & $\begin{array}{l}\text { forced mid-expira- } \\
\text { ratory flow: } l \cdot \mathrm{s}^{-1}\end{array}$ & $\begin{array}{l}\text { débit expiratoire } \\
\text { maximal médian: } \\
l \cdot \mathrm{s}^{-1}\end{array}$ & $\begin{array}{l}\text { maximaler mittlerer } \\
\text { exspiratorischer } \\
\text { Fluss: } l \cdot \mathrm{s}^{-1}\end{array}$ & $\begin{array}{l}\text { geforceerde mid- } \\
\text { expiratoire stroom: } \\
l \cdot \mathrm{s}^{-1}\end{array}$ & $\begin{array}{l}\text { forceret midteksspi- } \\
\text { torisk volumen- } \\
\text { strọm: } l \cdot \mathrm{s}^{-1}\end{array}$ & $\begin{array}{l}\text { flusso medio espira- } \\
\text { torio forzato nel tratto } \\
25-75 \% \text { della capacita } \\
\text { vitale forzata: } l \cdot \mathrm{s}^{-1}\end{array}$ & $\begin{array}{l}\text { flujo mesoespiratorio } \\
\text { forzado: } l \cdot \mathrm{s}^{-1}\end{array}$ & $\begin{array}{l}\text { débito expiratório } \\
\text { máximo médio: } l \cdot \mathrm{s}^{-1}\end{array}$ & 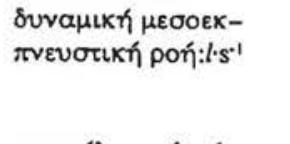 \\
\hline FEFV-curve & $\begin{array}{l}\text { forced expiratory } \\
\text { flow-volume curve }\end{array}$ & $\begin{array}{l}\text { courbe débit expi- } \\
\text { ratoire forcé-volume }\end{array}$ & $\begin{array}{l}\text { forcierte exspirato- } \\
\text { rische Fluss- } \\
\text { Volumen Kurve }\end{array}$ & $\begin{array}{l}\text { expiratoire stroom- } \\
\text { volume curve van } \\
\text { geforceerde uit- } \\
\text { ademing }\end{array}$ & $\begin{array}{l}\text { forceret eksspirato- } \\
\text { risk flow-volumen } \\
\text { kurve }\end{array}$ & $\begin{array}{l}\text { curva flusso-volume } \\
\text { espiratoria forzata }\end{array}$ & $\begin{array}{l}\text { curva de flujo-volumen } \\
\text { espiratoria forzada }\end{array}$ & $\begin{array}{l}\text { curva débito-volume } \\
\text { expiratória forçada }\end{array}$ & 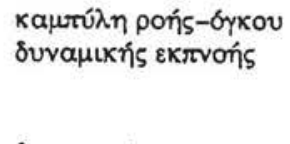 \\
\hline FES & $\begin{array}{l}\text { forced expiratory } \\
\text { spirogram }\end{array}$ & $\begin{array}{l}\text { spirogramme expi- } \\
\text { ratoire forcé }\end{array}$ & $\begin{array}{l}\text { forciertes exspira- } \\
\text { torisches Spiro- } \\
\text { gramm }\end{array}$ & $\begin{array}{l}\text { geforceerd expira- } \\
\text { toir spirogram }\end{array}$ & $\begin{array}{l}\text { forceret eksspirato- } \\
\text { risk spirogram }\end{array}$ & tracciato spirografico & espirograma forzado & $\begin{array}{l}\text { espirograma expiratorio } \\
\text { forçado }\end{array}$ & 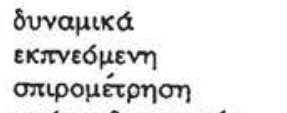 \\
\hline FET & $\begin{array}{l}\text { forced expiratory } \\
\text { time: } s\end{array}$ & $\begin{array}{l}\text { temps d'expiration } \\
\text { forcée: } s\end{array}$ & $\begin{array}{l}\text { forcierte exspirato- } \\
\text { rische Zeit: } s\end{array}$ & $\begin{array}{l}\text { geforceerde expi- } \\
\text { ratoire tijd: } s\end{array}$ & $\begin{array}{l}\text { forceret eksspira- } \\
\text { tionstid: s }\end{array}$ & $\begin{array}{l}\text { tempo espiratorio } \\
\text { forzato: } \mathrm{s}\end{array}$ & $\begin{array}{l}\text { tiempo espiratorio for- } \\
\text { zado: } s\end{array}$ & $\begin{array}{l}\text { tempo de expiração } \\
\text { forçada: } s\end{array}$ & 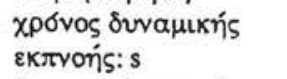 \\
\hline FEV, & $\begin{array}{l}\text { forced expiratory } \\
\text { volume in } t \\
\text { seconds: } l\end{array}$ & $\begin{array}{l}\text { volume expiratoire } \\
\text { force en } t \\
\text { secondes: } l\end{array}$ & $\begin{array}{l}\text { forciertes exspira- } \\
\text { torisches Volumen } \\
\text { in } t \text { Sekunden: } l\end{array}$ & $\begin{array}{l}\text { geforceerd expira } \\
\text { toir volume in } t \\
\text { seconden: } l\end{array}$ & $\begin{array}{l}\text { forceret eksspirato- } \\
\text { risk volumen it } \\
\text { sekunder: } l\end{array}$ & $\begin{array}{l}\text { volume espiratorio } \\
\text { forzato in } t \text { secondi: } l\end{array}$ & $\begin{array}{l}\text { volumen espiratorio } \\
\text { forzado en } t \text { segundos: } \\
l\end{array}$ & $\begin{array}{l}\text { volume expiratório for- } \\
\text { çado em } t \text { segundos: } l\end{array}$ & 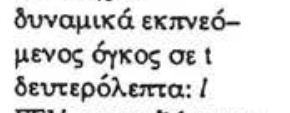 \\
\hline $\mathrm{FEV}, \% \mathrm{VC}$ & $\begin{array}{l}\text { FEV, as a percen- } \\
\text { tage of the vital } \\
\text { capacity (to be } \\
\text { specified) }\end{array}$ & $\begin{array}{l}\text { FEV, en pourcen- } \\
\text { tage de la capacite } \\
\text { vitale (à spécifier) }\end{array}$ & $\begin{array}{l}\text { FEV, als Prozent } \\
\text { der Vitalkapazität } \\
\text { (VC muss spezifi- } \\
\text { ziert werden) }\end{array}$ & $\begin{array}{l}\text { FEV, als percentage } \\
\text { van de vitale capa- } \\
\text { citeit (te specifice- } \\
\text { ren) }\end{array}$ & $\begin{array}{l}\text { FEV, angivet i pro- } \\
\text { cent af VC (speci- } \\
\text { ficeres) }\end{array}$ & $\begin{array}{l}\text { VEMS espresso in } \\
\text { percentuale della } \\
\text { capacità vitale } \\
\text { (da specificare) }\end{array}$ & $\begin{array}{l}\text { FEV, expresado como } \\
\text { porcentaje de la capaci- } \\
\text { dad vital (debe espifi- } \\
\text { carse) }\end{array}$ & $\begin{array}{l}\text { FEV em percentagem } \\
\text {-da capacidade vital (a } \\
\text { especificar) }\end{array}$ & 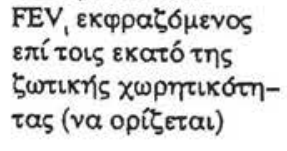 \\
\hline
\end{tabular}




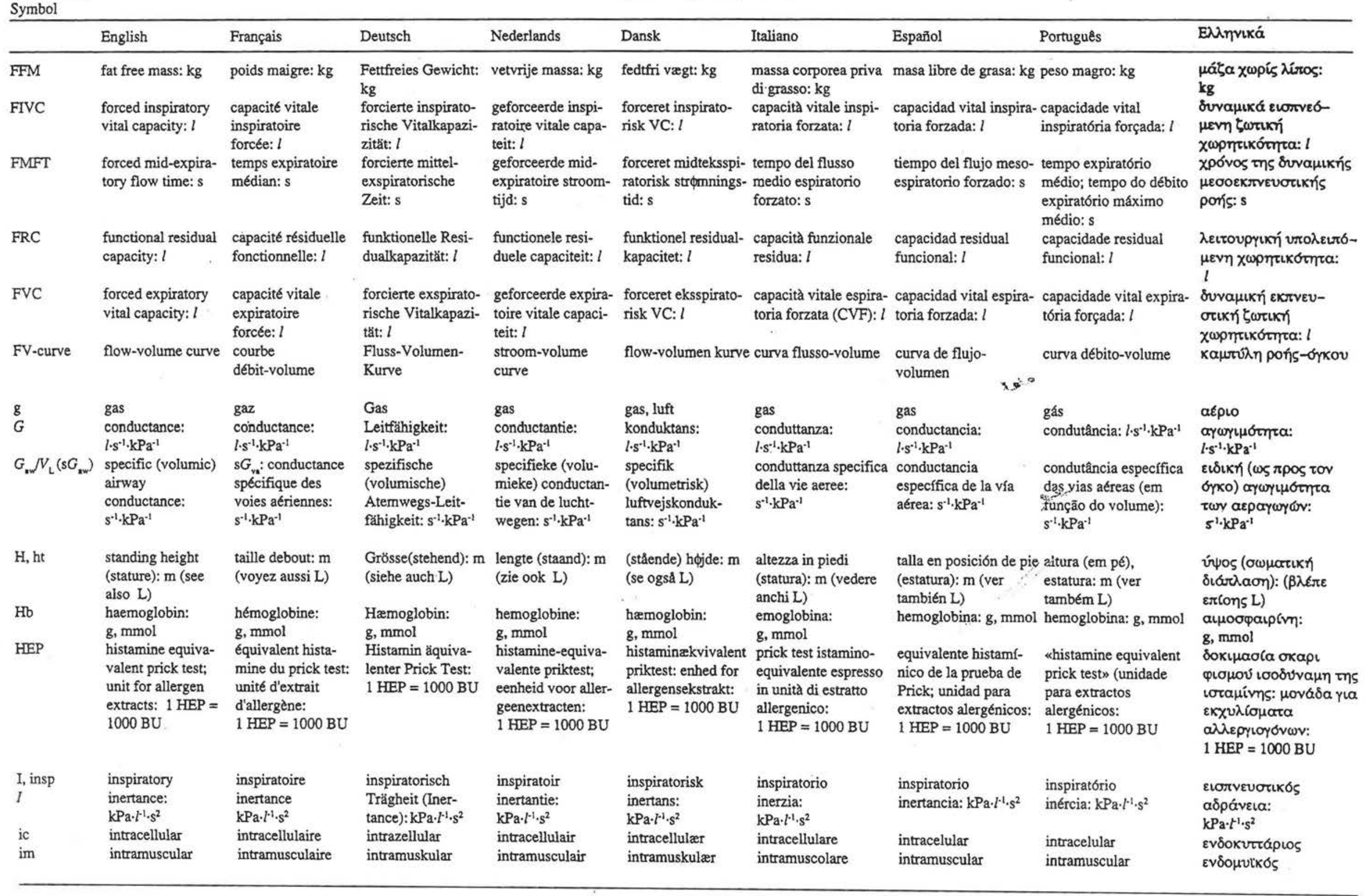


Português

Eג入ฑทนкó

\begin{tabular}{ll} 
& English \\
\hline IC & $\begin{array}{l}\text { inspiratory } \\
\text { capacity: } l\end{array}$ \\
IRV & inspiratory reserve \\
& volume: $l$ \\
it & intrathoracic \\
iv & intravenous \\
IVC & inspiratory vital \\
& capacity: $l$ \\
IVPF-curve & isovolume
\end{tabular}

Français

Dansk

Italiano

capacidad

capacidad inspiratoria: capacidade inspira-

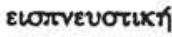

$\begin{array}{lll}\text { capacité } & \text { inspiratorische } & \text { inspiratoire } \\ \text { inspiratoire: } & \text { Kapazität: } l & \text { capaciteit: }\end{array}$

inspiratorisk

tória: $l$

$\begin{array}{ll}\text { volume de réserve } & \text { inspiratorisches } \\ \text { inspiratoire: } l & \text { Reservevolumen: } l\end{array}$

kapacitet: $l$ l

vol

tória:

volume de reserva

intrathorakal intrathoracaal intrathorakal intratoracico intratorácico

inspiratória:

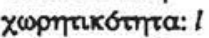

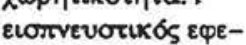

spuxos Grkos: $l$

Ev8̊otwpakıк6

intravenфs intravenoso intravenoso intravenoso

intravenös intraveneus

inspiratorisk VC: $l$ capacità vitale inspira-capacidad vital

capacité vitale

inspiratoire vi

capacidade vital

Vitalkapazităt: $l$ capaciteit: $l$

toria: $l \quad$ inspiratoria: $l$

sovolumen tryk-

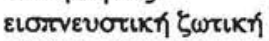

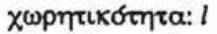

pressure-flow curve pression-déb

stroom curve

flow kurve

isovolume

curva flujo-presion

curva pressão-débito

каuтvi $\lambda$ porj-бyxov

isovolum

transfer

coefficient de

Transfer-

transfer coëfficiënt: transferkoefficient: coefficiente

$\mathrm{mmol} \cdot \mathrm{min}^{-1} \cdot \mathrm{kPa}^{-1} \cdot l^{-1} \mathrm{mmol} \cdot \mathrm{min}^{-1} \cdot \mathrm{kPa}^{-1} \cdot l^{-1}$ transfer:

coeficiente de

transferencia:

isovolume

cowv onkwe

$\mathrm{mmol} \cdot \mathrm{min}^{-1} \cdot \mathrm{kPa}^{-1} \cdot l^{-1} \mathrm{mmol} \cdot \mathrm{min}^{-1} \cdot \mathrm{kPa}^{-1} \cdot l^{-1} \mathrm{mmol} \cdot \mathrm{min}^{-1} \cdot \mathrm{kPa}^{-1}, l^{-1}$ (zie ook $T_{\mathrm{L}} / V_{\mathrm{A}}$ )

(se ogsa $T_{\mathrm{L}} / V_{\mathrm{A}}$ )

$\mathrm{mmol} \cdot \mathrm{min}$

coeficiente de difusão (ou de transferencia):

(see also $\left.T_{1} / V_{A}\right) \quad\left(\right.$ voyez aussi $\left.T_{L} / V_{A}\right) \quad$ (siehe auch $\left.T_{1} / V_{A}\right)$

longueur: $\mathrm{m}$;

Länge: $m$;

lengte: $m$;

litre

litre

lite

(vedere anche $T_{1} / V_{A}$ ) (ver también $T^{2} V_{N}$

(ver tambemin $T^{-1} \cdot \mathrm{kPa}^{-1} \cdot \mathrm{l}^{-1}$

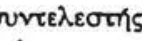

siáxuons:

$\mathrm{mmol} \cdot \mathrm{min}^{-1} \cdot \mathrm{kPa}^{-1} \cdot \gamma^{-1}$

$\left(\beta \lambda \varepsilon \pi \varepsilon \varepsilon \pi\right.$ lons $\left.T_{1} / N_{A}\right)$

standing height (sta- taille debout: $m \quad$ Grösse (stehend): $m$ lichaamslengte

ture): $\mathrm{m}$ (see also (voyez aussi $\mathrm{H}$ et (siehe auch $\mathrm{H}$ und (staand): $\mathrm{m}$ (zie

$\mathrm{H}$ and $\mathrm{ht}$ )

ht)

ht)

langde: $m$;

lunghezza: $\mathrm{m}$

longitud: $\mathrm{m}$;

comprimento: $\mathrm{m}$;

liter litro

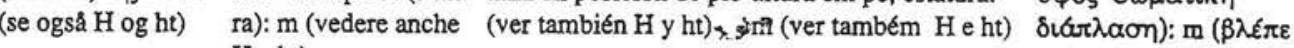

polmone

LAR

réaction asthma-

long

reaction

asthmatische

lunge

pulmón

pulmão

ertons $\mathrm{H}$ kat ht)

Spätreaktion reactie astmatisk

reazione asmatica

reacción asmática

reacção asmática tardia

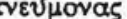

latéral

senreaktion

ritardata

tardía

lateraal

lateral

laterale

lateral

laminare

lateral

lamenter

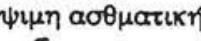

male

masculin

männlich

man

mandlig

membrane

masse: $\mathrm{kg}$

Masse: $\mathrm{kg}$

massa: $\mathrm{kg}$

maximal

maximal

maximal

maximaal

masse: $\mathrm{kg}$

membran

maschio, maschile

hombre

massa: $\mathrm{kg}$

masa: $\mathrm{kg}$

maksimal,

membrana

membran

maksimums-

massimo

máximo

masculino

massa; peso: $\mathrm{kg}$

mehrere Atemzüge multiple breath, meerdere adem.

multiple breath

respiro multiplo

respiración mựtitiple

maximo

multiples halingen

maximal expiratory débit expira-

maximaler exspira- maximale expira

respiraçōes múltiplas

averopaon

rixápios

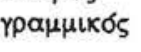

avopas

a.ta. kg

$\mu \varepsilon \mu \beta \rho \sigma r m$

$\mu E$ YLTTOS

$V_{\text {max }}$ )

(voyez aussi $V$.

. $1 \cdot \mathrm{s}^{-1}$ toire volumestroom: torisk volumen-

io mas- flujo espiratorio

débito expiratório

máximo: $l \cdot \mathrm{s}^{-1}$ (ver

também $V_{\max }$ )

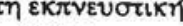

por: $1 \cdot \mathrm{s}^{-1}(\beta \lambda \varepsilon \pi \varepsilon$

$\varepsilon \pi$ (ons $V_{\text {.ux }}$ )

$V^{\prime}$ ) también $V_{\max }$ ) 


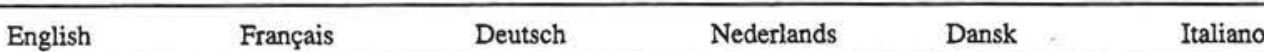

ano$$
\text { Español }
$$$$
\text { Português }
$$

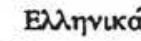

MEF $\quad$ MEF when $x \%$ of debitexpiraire the FVC $x \%$ of débit expiratoire be exhaled: $l \cdot \mathrm{s}^{-1}$ maximal lorsque

WEF wen $x \%$ der Manneer nog MEF i det $\phi j e b l i k$ (see also $V$

$\mathrm{x} \%$ de la FVC (voyez aussi $V_{\text {mes }}$ ) $\mathrm{x} \%$ van de FVC $\mathrm{x} \%$ af FVC endnu

flusso espiratorio

MEF cuand

el $x \%$

medio ad un determi- la FVC aún debes le di capacità vitale (ver también $V_{\text {mex }}^{\prime}$ )

forzata da espirare: $l \cdot \mathrm{s}^{-1}$

(vedere anche $V_{\max }$

ademd: $l \cdot \mathrm{s}^{-1}$ (zie ook $l \cdot \mathrm{s}^{-1}\left(\mathrm{se}\right.$ også $V_{\text {ma }}$ $\begin{array}{ll}\text { MEF }_{x \text { sTLC }} & \text { MEF when } x \% \text { of débit expiratoire } \\ \text { the TLC remains in maximal lorsque }\end{array}$ the lung: $l \cdot \mathrm{s}^{-1}$ (see $\mathrm{x} \%$ de la capacité also $V_{\max }^{\prime}$ ) totale reste dans poumon: $l \cdot s^{-1}$ (voyez aussi $V_{\text {max }}$ )

$\triangle M E F_{x a l}$ difference in MEF différence de MEF between iwo condi- entre deux con $x$ tions when $x \%$ of tions lorsque $x \%$ the FVC or TLC (to de la capacite exremains to be haled: $1 \cdot s^{-1}($ see de la capacité pulhaled: $7 \cdot s^{-1}(\mathrm{sec}$ monaire totale (a spécifier) reste à expirer: $l \cdot \mathrm{s}^{-1}$ (voyez aussi $\Delta V_{\text {, }}$ ) MEF wenn $\mathrm{x} \%$ der MEF wanneer nog MEF i det $\phi j$ jeblik TLC noch $\mathrm{x} \%$ van de TLC in $\mathrm{x} \%$ af TLC endnu dio ad un determinato

MEF quando falta MEF Grav $\times \%$ ms $\begin{array}{lll}\text { verbleiben: } l \cdot \mathrm{s}^{-1} & \text { de longen achter- } & \text { ikke er udândet: } \\ \left.\text { (siehe auch } V_{\text {max }}\right) & \text { blijft: } l \cdot \mathrm{s}^{-1}(\text { zie ook } & l \cdot \mathrm{s}^{-1}\left(\operatorname{se} \text { ogsa } V_{\text {max }}\right)\end{array}$ livello di capacità MEF cuando el $x \%$ de MEF quando resta nos

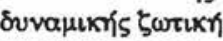

वro $\mu \varepsilon v \varepsilon เ v \alpha$

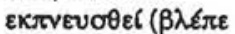

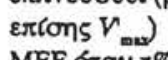
polmonare totale (vedere anche $V_{\text {mex }}$ ) en el pulmón: $l \cdot \mathrm{s}^{-1} \quad l \cdot \mathrm{s}^{-1}$ (ver também $V$

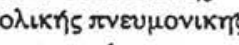
(ver tambien $V^{r}$ $V_{\max }$

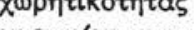

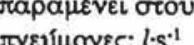
( $\beta \lambda \varepsilon \pi \varepsilon \varepsilon \pi i$ 们 $V_{\text {max }}$ )

Differenz in MEF zwischen zwei Bedingungen wenn TLC noch auszuatmen sind: $l \cdot \mathrm{s}^{-1}$ (siehe auch $\Delta V_{x}$ )

verschil in $V$ tus- forskel i MEF malt differenza di flusso wanneer no $x \%$ cuando el $x \%$ de la van de FVC of TLC $x \%$ af FVC eller tali, a determinati FVC o TLC (debe (te specificeren) TLC (specificeres) livelli percentuali di especificarse) aún moet worden uitge- endnu ikke er ademd: $l \cdot \mathrm{s}^{-1}$ (zie udåndet: $l \cdot \mathrm{s}^{-1}$ CVF espiratoria in rapporto alla CPT: rapporto alla CPT: $\Delta V_{\mathrm{x}}$ ) (ver también $\Delta V^{\prime}$, (se også $\Delta V$,

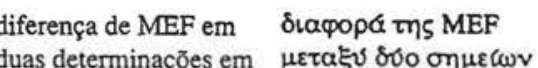

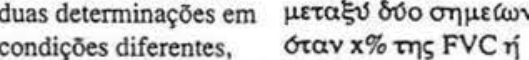

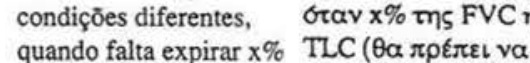

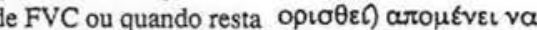

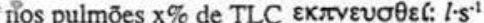
(a especificar): $l \cdot \mathrm{s}^{-1}$ (ver também $\Delta V$ )

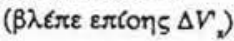
maximale exspirato- maximale expiramaximal expiratory courbe débit expr
flow-volume curve toire maximal$\begin{array}{ll}\text { toire maximal- } & \text { rische Fluss- } \\ \text { volume } & \text { Volumen-Kurve }\end{array}$ oommaksimal eksspira- curva flusso volume curva de flujo volumen curva débito-volume
torisk flow volumen espiratoria massima espiratoria máxima kurve

MIF $_{\text {xs:Fve }} \quad$ forced inspiratory forcierter inspiraflow when $x \%$ of inhaled: 1 forcé lorsque $\mathrm{x} \%$ de torischer Fluss, volume curve

flusso inspiratorio flujo inspiratorio forza- débito inspiratório

$\mu$ $\mu$ †

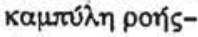

бyxor oire stroom als $x \%$ risk stromningshas- forzato quando $x \%$ do cuando el $x \%$ de la forcado quando $x \%$ de por orav $x \%$ tns VC

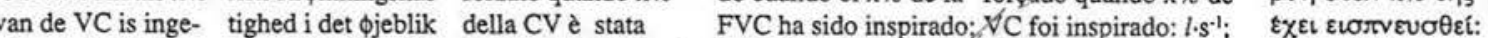
to be specified été inspiré: $l \cdot \mathrm{s}^{-1}$. VC à spécifier eingeatmet ist: $l \cdot s$ VC muss speziti-
ziert werden minimal minimal minimal specificeren minimaal

$l \cdot \mathrm{s}^{-1} ; \eta \mathrm{VC}$ va oplöri ceres)er inspiretet: specificare especificarse $l \cdot \mathrm{s}^{\mathrm{t}}$

$\min$ maximal mid-expi- débit expiratoire atory flow: $1 \cdot \mathrm{s}^{-1}$

maximal

maximaler mittelexspiratorischer Fluss: $l \cdot \mathrm{s}^{-1}$

mo mouth, buccal médian: $1 \cdot \mathrm{s}^{-1}$ Mund, Öffnung

minimo

mínimo

mínimo

$\varepsilon \lambda \alpha \times$ เ

maximale mid-expi- maksimal midteks- flusso medio-espirato- flujo máximo mesoratoire volumestroom: $l \cdot \mathrm{s}^{-1} \quad$ ningshastighed: $l \cdot \mathrm{s}^{-1}$ spiratorisk strom- ro massimo: $1 \cdot \mathrm{s}^{-1}$ espiratorio: $1 \cdot \mathrm{s}^{-1}$

débito expiratório

mond, buccaal mund, mundhule

boca, bucal musculaire muskulär mund, mundhule bocca, oral
muskel, muskulær muscular máximo médio: $l \cdot \mathrm{s}^{\cdot l}$ musculair

boca, bucal muscular

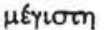

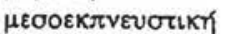
por): $\mid \cdot s^{-1}$

$\sigma \tau \mu \alpha, \sigma \tau о \mu \alpha \tau \kappa \kappa$ $\mu v t \times 65$ maximal voluntary ventilation maxi- maximale willkür- maximale vrijwillige maksimal volontær ventilazione massima ventilación voluntaria ventilação máxima ventilation at male volontaire breathing frequency à la frequence res- Atemfrequenz $\mathrm{f}$ : frequentie $\mathrm{f}: l \cdot \mathrm{min}^{-1}$ pirationsfrekvens terminata frequenza cia respiratoria f: respiratória f: $l \cdot \mathrm{min}^{-1}$ otos exoúotos

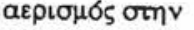
avarvevortKń ouxvórm 


\begin{tabular}{|c|c|c|c|c|c|c|c|c|c|}
\hline & English & Français & Deutsch & Nederlands & Dansk & Italiano & Español & Português & 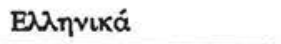 \\
\hline$n_{i}$ & $\begin{array}{l}\text { amount of } \\
\text { component i: mmol }\end{array}$ & $\begin{array}{l}\text { quantite du } \\
\text { composant i: mmol }\end{array}$ & $\begin{array}{l}\text { Menge einer Kom- } \\
\text { ponente i: } \mathrm{mmol}\end{array}$ & $\begin{array}{l}\text { hoeveelheid van } \\
\text { stof } \mathrm{i}: \mathrm{mmol}\end{array}$ & $\begin{array}{l}\text { mængde af } \\
\text { komponent i: mmol }\end{array}$ & $\begin{array}{l}\text { quantità millimolare } \\
\text { del componente i: } \\
\text { mmol }\end{array}$ & $\begin{array}{l}\text { cantidad del } \\
\text { componente i: mmol }\end{array}$ & $\begin{array}{l}\text { quantidade do } \\
\text { componente i: mmol }\end{array}$ & $\begin{array}{l}\text { roormta } \mu \text { Las } \\
\text { ovolas i: mmol }\end{array}$ \\
\hline$n_{1}^{\prime}$ & $\begin{array}{l}\text { instantaneous molar } \\
\text { flow of component } \\
\text { i: mmol } \mathrm{s}^{-1} \\
(\dot{n}, \text { permitted })\end{array}$ & $\begin{array}{l}\text { débit molaire du } \\
\text { composant i: } \\
\mathrm{mmol} \cdot \mathrm{s}^{-1} \\
\left(\dot{n}_{1} \text { permis }\right)\end{array}$ & $\begin{array}{l}\text { momentane molare } \\
\text { Strömung der Kom- } \\
\text { ponente i: } \mathrm{mmol} \cdot \mathrm{s}^{-1} \\
\left(\dot{n}_{i} \text { erlaubt) }\right.\end{array}$ & $\begin{array}{l}\text { momentane molaire } \\
\text { stroom van stof i: } \\
\text { mmol. } \mathrm{s}^{-1} \\
\left(\dot{n}_{1} \text { toegestaan }\right)\end{array}$ & $\begin{array}{l}\text { фjeblikkelig molar } \\
\text { stromningshastig- } \\
\text { hed af komponent } \\
\text { i: mmol } \mathrm{s}^{-1} \\
\text { ( } \dot{n}_{\mathrm{i}} \text { tilladt) }\end{array}$ & $\begin{array}{l}\text { flusso molare istan- } \\
\text { taneo del componente } \\
\text { i: mmol } \cdot \mathrm{s}^{-1} \\
\left(\dot{n}_{1} \text { consentito }\right)\end{array}$ & $\begin{array}{l}\text { flujo molar instantáneo } \\
\text { del componente i: } \\
\text { mmol } s^{-1} \\
\left(\dot{n}_{1} \text { permitido }\right)\end{array}$ & $\begin{array}{l}\text { débito molar instan- } \\
\text { tâneo do componente } \\
\text { i: mmol } \cdot \mathrm{s}^{-1} \\
\left(\dot{n}_{1} \text { permitido) }\right.\end{array}$ & 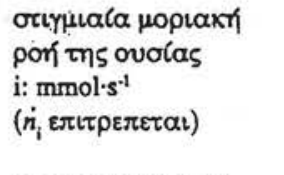 \\
\hline $\bar{n}_{1}^{\prime}$ & $\begin{array}{l}\text { molar transport of } \\
\text { component i: } \\
\text { mmol-min }-1 \\
\left(\dot{n}_{i} \text { permitted }\right)\end{array}$ & $\begin{array}{l}\text { débit molaire du } \\
\text { composant i: } \\
\text { mmol } \cdot \text { min }^{-1} \\
\left(\dot{n}_{1} \text { permis }\right)\end{array}$ & $\begin{array}{l}\text { Molartransport der } \\
\text { Komponente i: } \\
\text { mmol.min } \\
\left(\dot{n}_{1} \text { erlaubt }\right)\end{array}$ & $\begin{array}{l}\text { molair transport van } \\
\text { stof i: mmol-min } \\
\left(\dot{n}_{1} \text {, toegestaan }\right)\end{array}$ & $\begin{array}{l}\text { gennemsnitlig } \\
\text { molær strழmnings- } \\
\text { hastighed af kom- } \\
\text { ponent i: mmol-min } \\
\left(n_{1} \text { tilladt }\right)\end{array}$ & $\begin{array}{l}\text { trasporto molare di } \\
\text { componente i: } \\
\text { mmol-min }{ }^{-1} \\
\left.{ }^{(} \dot{n}_{\mathrm{i}} \text { consentito }\right)\end{array}$ & $\begin{array}{l}\text { transporte molar del } \\
\text { componente i: } \\
\text { mmol.min } \\
\left.\text { ( } \dot{n}_{1} \text { permitido }\right)\end{array}$ & $\begin{array}{l}\text { trasporte molar do } \\
\text { componente i: } \\
\text { mmol'min }{ }^{-1} \\
\left(\dot{n}_{1} \text { permitido }\right)\end{array}$ & 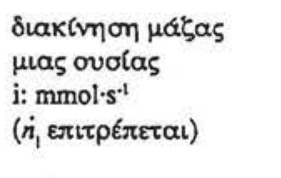 \\
\hline $\mathrm{N}_{2}$-slope & $\begin{array}{l}\text { slope of alveolar } \\
\text { plateau of expired } \\
\mathrm{N}_{2} \text { as a function of } \\
\text { exhaled volume } \\
\text { in } \mathrm{N}_{2} \text { single breath } \\
\text { test: } \% \mathrm{~N}_{2} \cdot l^{-1}\end{array}$ & $\begin{array}{l}\text { pente du plateau } \\
\text { alvéolaire d'azote } \\
\text { en fonction de volu- } \\
\text { me expiré dans le } \\
\text { test à l'azote en res- } \\
\text { piration unique: } \\
\% \mathrm{~N}_{2} \cdot l^{-1}\end{array}$ & $\begin{array}{l}\mathrm{N}_{2} \text {-Neigung: Nei- } \\
\text { gung des Alveolar- } \\
\text { plateaus entspre- } \\
\text { chend der Stick- } \\
\text { stofffraktion des } \\
\text { ausgeatmeten } \\
\text { Volumens beim } \\
\mathrm{N}_{2} \text {-Einatemzug- } \\
\text { test: } \% \mathrm{~N}_{2} \cdot l^{-1}\end{array}$ & $\begin{array}{l}\text { helling alveolair } \\
\text { plateau van de } \mathrm{N}_{2} \\
\text { «single breath" } \\
\text { test: } \% \mathrm{~N}_{2} \cdot l^{-1}\end{array}$ & $\begin{array}{l}\text { hældningen af fase } \\
\text { III i nitrogrammet: } \\
\% \mathrm{~N}_{2} \cdot l^{-1}\end{array}$ & $\begin{array}{l}\text { pendenza del plateau } \\
\text { alveolare dello azoto } \\
\text { espirato nel corso del } \\
\text { test di lavaggio del' } \\
\text { azoto in singolo } \\
\text { respiro: } \% \mathrm{~N}_{2} \cdot l^{-1}\end{array}$ & $\begin{array}{l}\text { pendiente alveolar de } \\
\mathrm{N}_{2} \text { espirado en función } \\
\text { del volumen espirado } \\
\text { en una prueba de nitro- } \\
\text { grafia de respiración } \\
\text { única: } \% \mathrm{~N}_{2} \cdot l^{-1}\end{array}$ & $\begin{array}{l}\text { inclinação (declive) da } \\
\text { fase alveolar no teste } \\
\text { de } \mathrm{N}_{2} \text { em respiração } \\
\text { - única em função do } \\
\text { volume expirado: } \\
\% \mathrm{~N}_{2} \cdot l^{-1}\end{array}$ & 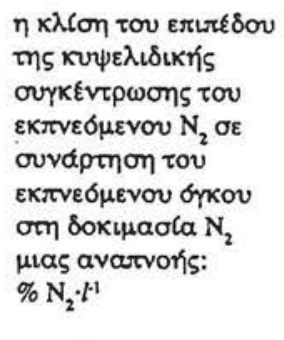 \\
\hline obs & observed & observé & beobachtet & waargenomen & observeret (mâlt) & osservato & observado & observado & 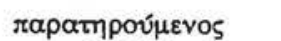 \\
\hline ODC & $\begin{array}{l}\text { oxyhaemoglobin } \\
\text { dissociation curve } \\
\text { oesophageal }\end{array}$ & $\begin{array}{l}\text { courbe de disso- } \\
\text { ciation de l'oxy- } \\
\text { hémoglobine } \\
\text { oesophagien }\end{array}$ & $\begin{array}{l}\text { Oxyhaemoglobin- } \\
\text { Dissoziationskurve } \\
\text { oesophagal }\end{array}$ & $\begin{array}{l}\text { oxyhemoglobine } \\
\text { dissociatiecurve } \\
\text { oesofagus }\end{array}$ & $\begin{array}{l}\text { oxyhæmoglobinets } \\
\text { dissociationskurve } \\
\text { oesofagus }\end{array}$ & $\begin{array}{l}\text { curva di dissociazione } \\
\text { dell'ossiemoglobina } \\
\text { esofageo }\end{array}$ & $\begin{array}{l}\text { curva de disociación } \\
\text { de la hemoglobina } \\
\text { esófago }\end{array}$ & $\begin{array}{l}\text { curva de dissociação } \\
\text { dớxihemoglobina } \\
\text { esofágico }\end{array}$ & 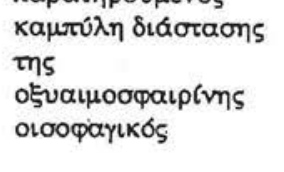 \\
\hline p & plasma & plasma & Plasma & plasma & plasma & plasma & plasma & plasma & $\pi \lambda \alpha \sigma \alpha \alpha$ \\
\hline$P$ & $\begin{array}{l}\text { pressure, } \\
\text { stress: } \mathrm{kPa}\end{array}$ & $\begin{array}{l}\text { pression, } \\
\text { contrainte: } \mathrm{kPa}\end{array}$ & Druck: $\mathrm{kPa}$ & druk, spanning: $\mathrm{kPa}$ & tryk, spæending: $\mathrm{kPa}$ & pressione, stress: $\mathrm{kPa}$ & presion, stress: $\mathrm{kPa}$. & pressão; força: $\mathrm{kPa}$ & rícon (stress) : $\mathrm{kPa}$ \\
\hline$P_{x, i}$ & $\begin{array}{l}\text { partial pressure of } \\
\text { component } \mathrm{i} \text { in } \\
\text { medium } \mathrm{x}: \mathrm{kPa}\end{array}$ & $\begin{array}{l}\text { pression partielle } \\
\text { du composant } \mathrm{i} \\
\text { dans le milieu } \mathrm{x} \text { : } \\
\mathrm{kPa}\end{array}$ & $\begin{array}{l}\text { Partialdruck der } \\
\text { Komponente } i \text { in } \\
x: \mathrm{kPa}\end{array}$ & $\begin{array}{l}\text { partiële druk van } \\
\text { stof } \mathrm{i} \text { in medium } \mathrm{x} \text { : } \\
\mathrm{kPa}\end{array}$ & $\begin{array}{l}\text { partialtryk af kom- } \\
\text { ponent } \mathrm{i} \text { i medium } \\
\mathrm{x}: \mathrm{kPa}\end{array}$ & $\begin{array}{l}\text { pressione parziale del } \\
\text { componente i nel } \\
\text { mezzo } x: \mathrm{kPa}\end{array}$ & $\begin{array}{l}\text { presion parcial del } \\
\text { componente i en el } \\
\text { medio } \mathrm{x}: \mathrm{kPa}\end{array}$ & $\begin{array}{l}\text { pressão parcial do } \\
\text { componente i no meio } \\
\mathrm{x}: \mathrm{kPa}\end{array}$ & 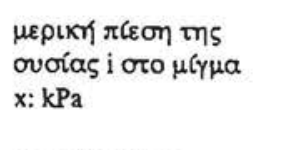 \\
\hline $\mathrm{PC}_{x}$ & $\begin{array}{l}\text { provocative con- } \\
\text { centration of bron- } \\
\text { choconstrictor cau- } \\
\text { sing FEV, to fall } \mathrm{x} \% \\
\text { from baseline: } \\
\mathrm{mg} \cdot \mathrm{ml}^{-1}, \text { mmol } l^{-1}\end{array}$ & $\begin{array}{l}\text { concentration } \\
\text { d'agent bronchocon- } \\
\text { stricteur provoquant } \\
6 \text { une chute du VEMS } \\
\text { de } x \% \text { de la valeur } \\
\text { de base: } \mathrm{mg}^{-1} \mathrm{ml}^{-1} \text {, } \\
\mathrm{mmol} \cdot \mathrm{ml} l^{-1}\end{array}$ & $\begin{array}{l}\text { Konzentration einer } \\
\text { bronchokonstricto- } \\
\text { rischen Substanz, } \\
\text { die einen } 20 \% \text {-igen } \\
\text { Abfall des FEV, } \\
\text { verursacht: } \mathrm{mg}^{-\mathrm{ml}^{-1}} \text {, } \\
\mathrm{mmol} \cdot \mathrm{ml}^{-1}\end{array}$ & $\begin{array}{l}\text { concentratie van } \\
\text { bronchusvernauwer } \\
\text { die } \mathrm{FEV}_{1} \text { met } \mathrm{x} \% \\
\text { doet dalen t.o.v. } \\
\text { uitgangswaarde: } \\
\mathrm{mg} \cdot \mathrm{ml}^{-1}, \mathrm{mmol} \cdot \gamma^{-1}\end{array}$ & $\begin{array}{l}\text { bronkieforsnæviende } \\
\text { koncentration, der } \\
\text { bevirker et fald i } \\
\text { FEV, p\&̊ x\% af } \\
\text { udgangsværdien: } \\
\text { mg } \cdot \mathrm{ml}^{-1}, \text { mmol } \cdot l^{-1}\end{array}$ & $\begin{array}{l}\text { econcentrazione di } \\
\text { agente broncoostrut- } \\
\text { tore in grado di provo- } \\
\text { care una variazione } \mathrm{x} \\
\text { dell' indice di misura } \\
\text { della risposta bronco- } \\
\text { ostruttiva: } \mathrm{mg} \cdot \mathrm{ml}^{-1} \text {, } \\
\mathrm{mmol} \cdot \mathrm{l}^{-1}\end{array}$ & $\begin{array}{l}\text { concentración de } \\
\text { agente broncoconstric- } \\
\text { - tor causante de una } \\
\text { cafda del FEV, basal } \\
\text { del } x \%: \text { mg } \cdot \mathrm{ml}^{-1} \text {, } \\
\text { mmol } \cdot l^{-1}\end{array}$ & $\begin{array}{l}\text { concentração de agente } \\
\text { broncoconstritor que } \\
\text { causa a descida de } \mathrm{x} \% \\
\text { do valor inicial de } \mathrm{FEV}, \text {; } \\
\mathrm{mg} \cdot \mathrm{ml}^{-1}, \mathrm{mmol} \cdot l^{-1}\end{array}$ & 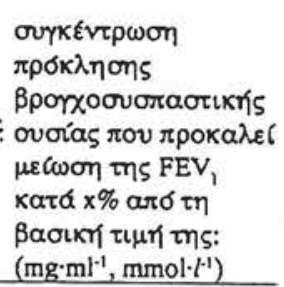 \\
\hline
\end{tabular}


provocative dose of bronchoconstrictor causing $\mathrm{FEV}_{1}$ to fall $\mathrm{x} \%$ from baseline: $\mathrm{mg}, \mu \mathrm{mol}$

dose d'agent bron- Dosis einer bron- dosis van bronbronkieforsnæychoconstricteur chokonstrictorischen chusvernauwer provoquant une Substanz, die einen die FEV, met $x \%$ chute de VEMS de $20 \%$-igen Abfall des doet dalen t.o.v. $\mathrm{x} \%$ de la valeur de FEV, verursacht: uitgangswaarde: base: $\mathrm{mg}, \mu \mathrm{mol}$ FEV, verursacht: bronkieforsnæv bevirker et fald $i$ $\mathrm{FEV}_{1} \mathrm{pa} \times \%$ af udgangsværdien $\mathrm{mg}$, mmol

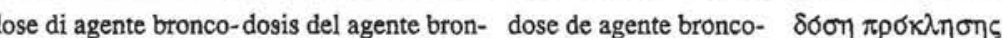

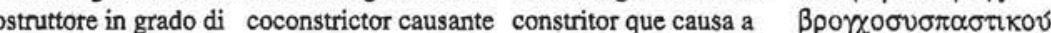

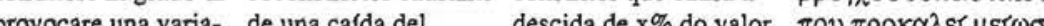
provocare una aria de una cafda del

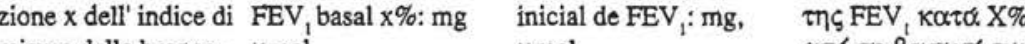
misura della bronco- $\mu \mathrm{mo}$ $\mu \mathrm{mol}$ $\alpha \pi \delta \tau \eta \beta \alpha \sigma \kappa \pi) \tau \mu \eta$ tाs: $\mathrm{mg}, \mu \mathrm{mol}$ débit expiratoire exspiratorischer expiratoire piek- eksspiratorisk peruzione. mg, $\mu \mathrm{mol}$

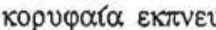
flow: $1 \cdot \mathrm{s}^{-1}, 1 \cdot \mathrm{min}^{-1}$ de pointe: $l \cdot s^{-1}$. Spitzenfluss: $l \cdot \mathrm{s}^{-1}$, stroom: $l \cdot \mathrm{s}^{-1}, l \cdot \mathrm{min}^{-1}$ picco di flusso espira-

peakflow (spidsvolumenstrom): $l \cdot \mathrm{s}^{: 1}$,

$l \cdot \mathrm{min}^{-1}$

PEFV curve partial expiratory courbe débit flow-volume curve expiré-volume l.min

partielle exspirato- partiële expiratoire forceret eksspirato- curva flusso-volume curva de flujorische Fluss- stroom-volume risk flow-volumen espiratoria parziale volumen parcia Volumen Kurve curve kurve met start ved mindre end maximal partielle indåndning

unit of acidity unité d'acidité

Einheit der Säure

eenheid van zuur-

unità di acidità unidad de ácidez

fysiologisk fisiologico fisiologico

physiologisch physiological physiologique de pointe: $l \cdot \mathrm{s}^{-1}$, graad

$\begin{array}{ll}\text { phys } & \text { physiological } \\ \text { PIF } & \text { peak inspiratory } \\ & \text { flow: } l \cdot \mathrm{s}^{-1}, l \cdot \mathrm{min}^{-1}\end{array}$ min $^{-1}$ inspiratorischer Spitzenfluss: $l \cdot \mathrm{s}^{-1}, l \cdot \mathrm{min}^{-1}$

fysiologisch

stroom; $l \cdot \mathrm{s}^{-1}, l \cdot \mathrm{min}^{-1}$ flow (spidsvolumen- torio: $l \cdot \mathrm{s}^{-1}, l \cdot \mathrm{min}^{-1}$ ratorio: $l \cdot \mathrm{s}^{-1}, l \cdot \mathrm{min}^{-1}$ strøm): $l \cdot \mathrm{s}^{-1}, l \cdot \mathrm{min}^{-1}$

pleural pleural pleural pleuraal pleura

pred predicted préd; prédit

vorhergesagt predicted pulmonaire

pulmonal

voorspeld

pleurico pletismografico eregnet (reference-)

pulm pulmonary

blood volume: $l$ olume sanguin: $l$ Blutvolumen: pulmonaal lunge

teorico, predetto

pleural

pletismográfico

teórico, de referencia

débito expiratório

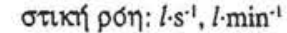
$\mathrm{s}^{-1}, \mathrm{~min}$

curva débito-volume expiratória parcial

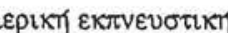
$\kappa \alpha \mu \pi v \lambda \eta$ poris-бүкоv instantaneous blood débit sanguin momentaner pulmonar

unidade de acidez

$\mu \circ v \alpha \delta \alpha, 0 \xi \sigma \tau \eta \tau, \varsigma$

fisiologico

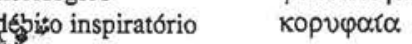

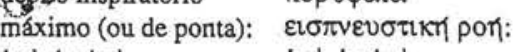
l. $\mathrm{s}^{-1}, l \cdot \mathrm{min}^{-1}$ pleural $\cdot \mathrm{s}^{-1}, l \cdot \mathrm{min}^{-1}$
$\pi \lambda \varepsilon v \cos \sigma \mathrm{s}$ $\pi \lambda \eta \theta v \sigma \mu о$ ора.чікбе $\pi \rho \circ \beta \lambda \varepsilon \pi \delta \mu \varepsilon v \circ \varsigma$

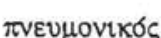

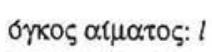

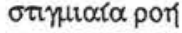
$\alpha \tau \mu \alpha \tau \circ \varsigma: l \cdot s^{-1}$ volumenstr $\phi \mathrm{m}$ (blod): $l \cdot \mathrm{s}^{-1}$

volume ematico: $l$ istantaneo: $l \cdot \mathrm{s}^{-1}$

volumen de sangre: $/$ pulmonar

instantáneo: $l \cdot \mathrm{s}^{-1}$ débito sangứneo instantaneo: $l \cdot \mathrm{s}^{-1}$

volumeversnelling volumenaccelera-

Blutvolumen-

lood volume

accélération

Beschleunigun l.s.-2 tion (blod): $l \cdot \mathrm{s}^{-2}$

accelerazione del

aceleración del

aceleração do volume

volume ematico: $l \cdot \mathrm{s}^{-2}$ volumen sanguíneo: sanguíneo: $l \cdot \mathrm{s}^{-2}$ $l \cdot s^{-2}$ time-averaged débit sanguin mittleres Herz- tijdgemiddelde time-averaged (perfusion): $l \cdot \mathrm{min}^{-1} \quad l \cdot \mathrm{min}$ zeitvolumen bloedstroom

gennemsnitlig $\mathrm{s}^{-2}$

gennemblodningshastighed: $l \cdot \mathrm{min}^{-1}$ $(\dot{Q}$ erlaubt) ( $)$ toegestaan $) \quad(\dot{Q}$ tilladt) nel temin

débito sanguíneo méd

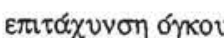

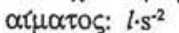

por $\alpha\{\mu \alpha \tau \cos \omega$ po hjertets minut l. $\mathrm{min}^{-1}$ (Q permitido) (Q́ permitido)

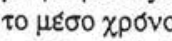
$(\alpha \mu \alpha \alpha \tau \omega \sigma): l \cdot \min ^{-1}$ (Q: $\varepsilon \pi \tau \tau \rho \in \pi \varepsilon \tau \alpha)$

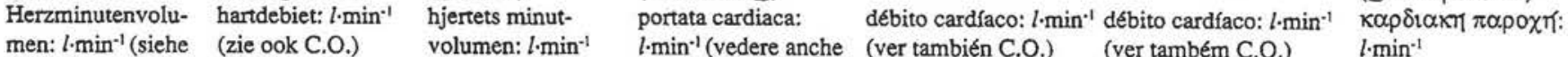
(se også C.O.) C.O.)

-min $\begin{array}{lll}l \cdot \mathrm{min}^{-1}(\text { voyez } & \text { auch C.O.) }\end{array}$

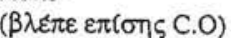

strфmningsmodstand: $\mathrm{kPa} \cdot l^{-1} \cdot \mathrm{s}$ stromingsweer$\begin{array}{lll}\begin{array}{l}\text { résistance al l'écou- } \\ \text { lement: } \mathrm{kPa} \cdot l^{1} \cdot \mathrm{s}\end{array} & \text { Strömungswider } \mathrm{kPa} \cdot l^{1} \cdot \mathrm{s}\end{array}$ stand: $\mathrm{kPa} \cdot l^{-1} \cdot \mathrm{s}$

\section{resistenza al flusso:
$\mathrm{kPa} \cdot l^{-1} \cdot \mathrm{s}$} $\mathrm{kPa} \cdot l^{-1} \cdot \mathrm{s}$ resistência ao fluxo: $\mathrm{kPa} \cdot l^{1 \mathrm{t}}$

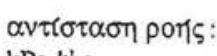
$\mathrm{kPa} \cdot \mathrm{l}^{-1} \cdot \mathrm{s}$ 


\begin{tabular}{|c|c|c|c|c|c|c|c|c|c|}
\hline & English & Français & Deutsch & Nederlands & Dansk & Italiano & Español & Portuguess & 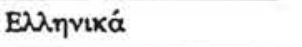 \\
\hline$R$ & $\begin{array}{l}\text { respiratory } \\
\text { quotient: dimen- } \\
\text { sionless }\end{array}$ & $\begin{array}{l}\text { quotient respira- } \\
\text { toire: sans dimen- } \\
\text { sion }\end{array}$ & $\begin{array}{l}\text { respiratorischer } \\
\text { Quotient: dimen- } \\
\text { sionslos }\end{array}$ & $\begin{array}{l}\text { respiratoire gas- } \\
\text { wisselingsverhou- } \\
\text { ding: dimensie- } \\
\text { loos }\end{array}$ & $\begin{array}{l}\text { respiratorisk } \\
\text { kvotient; fraktion: } \\
\text { dimensionslфs }\end{array}$ & $\begin{array}{l}\text { quoziente respiratorio: } \\
\text { privo di dimensione }\end{array}$ & $\begin{array}{l}\text { : cociente respiratorio: } \\
\text { sin dimensiones }\end{array}$ & $\begin{array}{l}\text { quociente respiratório: } \\
\text { sem unidade de medida }\end{array}$ & 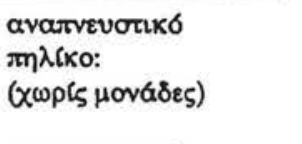 \\
\hline $\begin{array}{l}R \\
\mathrm{rb}\end{array}$ & $\begin{array}{l}\text { respiratory } \\
\text { rebreathing }\end{array}$ & $\begin{array}{l}\text { respiratoire } \\
\text { réinspiration }\end{array}$ & $\begin{array}{l}\text { respiratorisch } \\
\text { Rúckatmung }\end{array}$ & $\begin{array}{l}\text { respiratoir } \\
\text { terugademing }\end{array}$ & $\begin{array}{l}\text { respiratorisk } \\
\text { genândings- }\end{array}$ & $\begin{array}{l}\text { respiratorio } \\
\text { rirespirazione }\end{array}$ & $\begin{array}{l}\text { respiratorio } \\
\text { reinspiración }\end{array}$ & $\begin{array}{l}\text { respiratório } \\
\text { reinspiração }\end{array}$ & 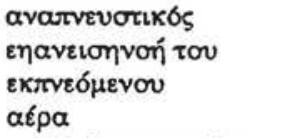 \\
\hline $\begin{array}{l}\text { RBC } \\
\text { RHE }\end{array}$ & $\begin{array}{l}\text { red blood cell } \\
\text { respiratory heat } \\
\text { exchange: } \mathrm{kJ} \cdot \mathrm{min}^{-1}\end{array}$ & $\begin{array}{l}\text { globule rouge } \\
\text { échange de chaleur } \\
\text { respiratoire: } \\
\mathrm{kJ} \cdot \mathrm{min}^{-1}\end{array}$ & $\begin{array}{l}\text { rote Blutzelle } \\
\text { respiratorischer } \\
\text { Wärmeaustausch } \\
\mathrm{kJ} \cdot \mathrm{min}^{-1}\end{array}$ & $\begin{array}{l}\text { rode bloedcel } \\
\text { respiratoire } \\
\text { warmte-uitwisse- } \\
\text { ling: } \mathrm{k} \cdot \min ^{-1}\end{array}$ & $\begin{array}{l}\text { rфde blodlegemer } \\
\text { respiratorisk } \\
\text { varmeudveksling: } \\
\mathrm{kJ} \cdot \mathrm{min}^{-1}\end{array}$ & $\begin{array}{l}\text { globuli rossi } \\
\text { scambio calorico } \\
\text { respiratorio: } \mathrm{kJ} \cdot \mathrm{min}^{-1}\end{array}$ & $\begin{array}{l}\text { hematfe } \\
\text { intercambio } \\
\text { respiratorio de } \\
\text { calor: } \mathrm{kJ} \cdot \mathrm{min}^{-1}\end{array}$ & $\begin{array}{l}\text { glóbulo vermelho } \\
\text { troca respiratória de } \\
\text { calor: } \mathrm{kJ} \cdot \mathrm{min}^{-1}\end{array}$ & 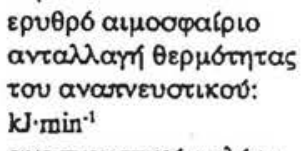 \\
\hline RQ & $\begin{array}{l}\text { respiratory } \\
\text { quotient: dimen- } \\
\text { sionless }\end{array}$ & $\begin{array}{l}\text { quotient } \\
\text { respiratoire: sans } \\
\text { dimension }\end{array}$ & $\begin{array}{l}\text { respiratorischer } \\
\text { Quotient; Atemgas- } \\
\text { austauschverhält- } \\
\text { nis: dimensionslos }\end{array}$ & $\begin{array}{l}\text { respiratoir quo- } \\
\text { tient: dimensieloos }\end{array}$ & $\begin{array}{l}\text { respiratorisk } \\
\text { kvotient; fraktion: } \\
\text { dimensionslфs }\end{array}$ & $\begin{array}{l}\text { quoziente respiratorio: } \\
\text { privo di dimensione }\end{array}$ & $\begin{array}{l}\text { cociente respiratorio: } \\
\text { sin dimensiones }\end{array}$ & $\begin{array}{l}\text { quociente respiratório: } \\
\text { sem unidade de medida }\end{array}$ & 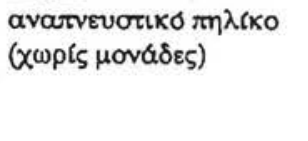 \\
\hline rs & respiratory system & $\begin{array}{l}\text { système } \\
\text { respiratoire }\end{array}$ & $\begin{array}{l}\text { respiratorisches } \\
\text { System }\end{array}$ & $\begin{array}{l}\text { respiratoir } \\
\text { systeem }\end{array}$ & $\begin{array}{l}\text { respiratoriske } \\
\text { system }\end{array}$ & sistema respiratorio & sistema respiratorio & sistema respiratório & 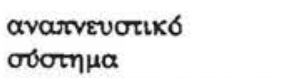 \\
\hline RV & residual volume: $l$ & $\begin{array}{l}\text { volume } \\
\text { résiduel: } l\end{array}$ & $\begin{array}{l}\text { Residualvolumen: } \\
l\end{array}$ & residuele volume: $l$ & residualvolumen: $l$ & volume residuo: $l$ & volumen residual: $l$ & volume residual: $l$ & 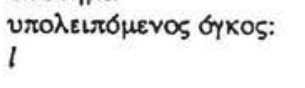 \\
\hline $\mathrm{s}$ & second & seconde & Sekunde & seconde & sekund & secondo & segundo & segundo & 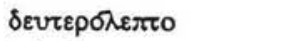 \\
\hline$s$ & specific & spécifique & spezifisch & specifiek & specifik & specifico & específico & especffico & $\varepsilon เ \delta\llcorner K \measuredangle \zeta$ \\
\hline$S_{x, 1}$ & $\begin{array}{l}\text { saturation of com- } \\
\text { ponent } i \text { in medium } \\
x \text { : dimensionless }\end{array}$ & $\begin{array}{l}\text { saturation du com- } \\
\text { posant i dans le } \\
\text { milieu x: sans } \\
\text { dimension }\end{array}$ & $\begin{array}{l}\text { Sättigung der Kom- } \\
\text { ponente i in } \mathrm{x}: \\
\text { dimensionslos }\end{array}$ & $\begin{array}{l}\text { verzadiging met } \\
\text { substantie } \mathrm{i} \text { in } \\
\text { milieu } \mathrm{x} \text { : dimen- } \\
\text { sieloos }\end{array}$ & $\begin{array}{l}\text { matningsgrad af } \\
\text { komponent } \mathrm{i} \text { i } \\
\text { medium } \mathrm{x} \text { (fraktion) } \\
\text { dimensionsil } \phi \mathrm{s}\end{array}$ & $\begin{array}{l}\text { saturazione del com- } \\
\text { ponente i nell'elemen- } \\
\text { to } x \text { : privo di } \\
\text { dimensione }\end{array}$ & $\begin{array}{l}\text { saturación del compo- } \\
\text { nente i en el medio } x \text { : } \\
\text { sin dimensiones }\end{array}$ & $\begin{array}{l}\text { saturaç do } \\
\text { componente i no meio } \\
x: \text { sem unidade de } \\
\text { medida }\end{array}$ & 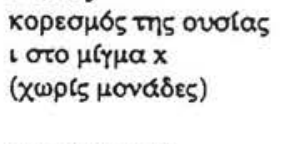 \\
\hline$s b$ & single breath & respiration unique & Einatemzug & $\begin{array}{l}\text { single breath; } \\
\text { enkele ademhaling }\end{array}$ & enkelt ândedrats- & respiro singolo & respiración única & respirzação única & $\mu$ на avarvon \\
\hline sc & subcutaneous & sous-cutané & subcutan & subcutaan & subkutan & sottocutaneo & subcútaneo & 'subcutâneo & vтоסбptos \\
\hline SH & sitting height: $\mathrm{m}$ & taille assise: $\mathrm{m}$ & Sitzhöhe: $\mathrm{m}$ & zithoogte: $\mathrm{m}$ & siddende hфjde: $\mathrm{m}$ & statura da seduti: $m$ & $\begin{array}{l}\text { talla en posición } \\
\text { sentada: } m\end{array}$ & altura (sentado): $\mathrm{m}$ & $\begin{array}{l}v \psi \propto \zeta \kappa \alpha \theta \eta \mu \varepsilon v \sigma v \\
\alpha \tau \sigma \mu \sigma v\end{array}$ \\
\hline $\begin{array}{l}\text { sh } \\
\text { sp }\end{array}$ & $\begin{array}{l}\text { shunt } \\
\text { spirometric }\end{array}$ & $\begin{array}{l}\text { shunt } \\
\text { spirométrique }\end{array}$ & $\begin{array}{l}\text { Shunt } \\
\text { spirometrisch }\end{array}$ & $\begin{array}{l}\text { shunt } \\
\text { spirometrisch }\end{array}$ & $\begin{array}{l}\text { shunt } \\
\text { spirometer, } \\
\text { spirometri- }\end{array}$ & $\begin{array}{l}\text { shunt } \\
\text { spirometrico }\end{array}$ & $\begin{array}{l}\text { cortocircuito } \\
\text { espirométrico }\end{array}$ & $\begin{array}{l}\text { "shunt", curto-circuito } \\
\text { espirométrico }\end{array}$ & 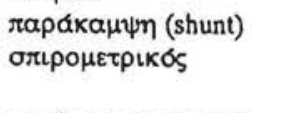 \\
\hline ss & steady state & état stable & Steady State & evenwichtstoestand & "steady state" & regime stabile & estado estacionario & $\begin{array}{l}\text { estado estabilizado; } \\
\text { regime estavel }\end{array}$ & 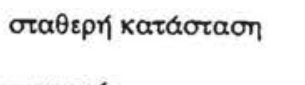 \\
\hline st & static & statique & statisch & statisch & statisk & statico & estático & estático & $\sigma \tau \alpha \tau ı x \sigma s$ \\
\hline STPD & $\begin{array}{l}\text { standard tempera- } \\
\text { ture and pressure, } \\
\text { dry }\end{array}$ & $\begin{array}{l}\text { pression et tempe- } \\
\text { rature standard, } \\
\mathrm{sec}\end{array}$ & $\begin{array}{l}\text { Standard-Tempera- } \\
\text { tur, Druck, und } \\
\text { trocken }\end{array}$ & $\begin{array}{l}\text { standaard tempera- } \\
\text { tuur en druk, droog }\end{array}$ & $\begin{array}{l}\text { standard temperatur } \\
\text { og tryk, t } \phi \mathrm{r}\end{array}$ & $\begin{array}{l}\text { temperatura e pres- } \\
\text { sione standard, secco }\end{array}$ & $\begin{array}{l}\text { temperatura y presión } \\
\text { barométrica estandar } \\
\text { sin humedad }\end{array}$ & $\begin{array}{l}\text { temperatura e pressão } \\
\text { estandardizadas, seco }\end{array}$ & 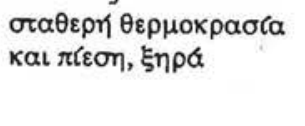 \\
\hline$t$ & $\begin{array}{l}\text { Celsius tempera- } \\
\text { ture: }{ }^{\circ} \mathrm{C}\end{array}$ & $\begin{array}{l}\text { temperature Celsius: } \\
{ }^{\circ} \mathrm{C}\end{array}$ & $\begin{array}{l}\text { Celsius Temperatur: } \\
{ }^{\circ} \mathrm{C}\end{array}$ & $\begin{array}{l}\text { Celsius } \\
\text { temperatuur: }{ }^{\circ} \mathrm{C}\end{array}$ & temperatur: ${ }^{\circ} \mathrm{C}$ & $\begin{array}{l}\text { temperatura in gradi } \\
\text { centigradi: }{ }^{\circ} \mathrm{C}\end{array}$ & $\begin{array}{l}\text { temperatura en grados } \\
\text { Celsius: }{ }^{\circ} \mathrm{C}\end{array}$ & $\begin{array}{l}\text { temperatura Celsius: } \\
{ }^{\circ} \mathrm{C}\end{array}$ & 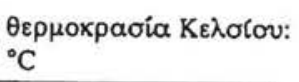 \\
\hline
\end{tabular}




English Français Nederlands Dansk

Italiano

Español

Português

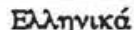

time: s; permitted: temps: s; permis: Zeit: s; exlaubt: minute, hour, day, minute, heure, jour, Minute, Stunde, year annee Tag, Jahr

tijd: s; toegestaan: tid: $s$; tilladt: minut, tempo: $s$; permesso in tiempo: s: permitido: tempo: $s$; permitidos: minuut, uur, dag, time, dag) Ar minuti, ore, giomi, minuto, hora, dfa, año minuto, hora, dia, ano ja

$\begin{array}{ll}\text { temperature thermo- thermodynamische } \\ \text { temperature: } \mathrm{K} & \text { dynamique: } \mathrm{K} \text { Temperatur: } \mathrm{K}\end{array}$

anni

tidal

$$
\text { courant }
$$

Atemzug. (Tidal)

temperatuur: $\mathrm{K}$

termodynamisk

emperatura termodi- temperatura termo-

temperatur

"tidal"; op- en

ratur: $\mathrm{K}$

namica: $K$

dinámica: $\mathrm{K}$

termodinâmica: $\mathrm{K}$

corrente

\section{neergaand, teug}

as transfer factor facteur de transfert Gastransferfaktor

transfer faktor for fattore di transfer

for the lung: gazeux pour le der Lunge:

voor de long:

corriente

factor de transferencia factor de difusão

gassoso polmonare: para el pulmón: (transferência) gasosa $\mathrm{mmol} \cdot \mathrm{min}^{-1} \cdot \mathrm{kPa}^{-1}$

$\mathrm{mmol} \cdot \mathrm{min}^{-1} \cdot \mathrm{kPa}^{-1}$

do pulmão: $\mathrm{mmol} \cdot \mathrm{min}^{-1} \cdot \mathrm{kPa}^{-1}$

coeficiente de transfe- coeficiente de difusẫo

transfer coëfficiënt: transferkoefficient: coefficiente di

rencia (ver $K$ )

(ou de transferencia)

$$
\text { (ver } K \text { ) }
$$

expiratoire adem- eksspirations-

tijd: $s$

eksspirations-

duración de la

duracäo da

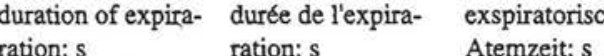

duration of inspira- duree de l'inspira- inspiratorische

duration of 1

inspiratorische inspiratoire adem- inspiration

$\begin{array}{ll}\text { inspiratoire adem- inspiration } \\ \text { tijd: } s & \text { længde: } s\end{array}$

totale ademtijd: $s$ respiratorisk

tempo inspiratorio:

duration of total durée de cycle res- totale Atemzy-

kluszeit: s

cykluslængde:

tempo totale del

espiracion:

duración de la

tempo expiratório: $s$ inspiración: $s$ tempo inspiratório: $s$

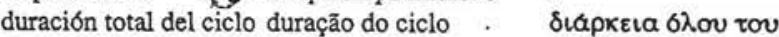

ciclo espiratorio: $\mathrm{s}$

respiratorio: s respiratório total; tempo avarvevortxớ

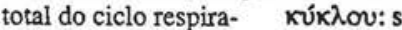

tório: $s$

TD

transcutaneous

anscutane

transkutan transcutaan transkutan

Toluen Diisocyanat tolueen diisocyanaat

transcutaneo

transcutáneo

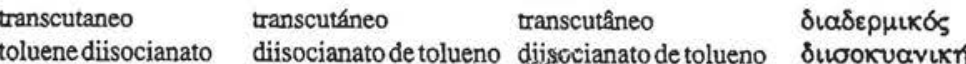

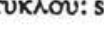

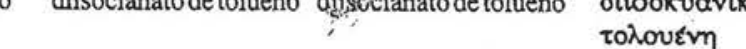

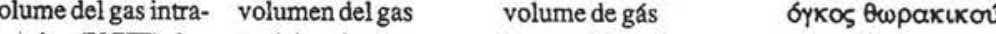

toracico (VGT): $l$ torácico:l intratorácico:l

toracico

tessuto

toŕcico

volumen:l

volume: $l$

intrathorakalt

thorax, thorakal

thoracic thoracique

total lung capacity: capacité pulmo-

Gewebe

weefsel

totale Lungen- totale longcapa- totallungekapa-

capacità polmonare

totale (CPT): $l$

citeit: $l$

transmural

total

naire totale: $l \quad$ kapazität: $l$

transmura

transpulmonary transmural

trachea

transrespiratory
transthoracic

turbulent

transpulmonaire

trachée

total

transmuraal

transpulmonal

totaal

total lungekapa-

citet: !

total

transmurale

totale

transpulmonal

transpulmonaal

trachea

trachea

transpolmonare

trachea

tejido

tecido

atpa: $l$

transrespiratorisch transrespiratoir transrespiratorisk

transthoracique

transthorakal transthoracaal

transthorakal

transrespiratorio

transtoracico

turbulent

transtoracico

tidal volume:

volume courant:

turbulent

$\left(\right.$ see also $V_{\mathrm{T}}$ )

Atemzugsvolumen: ademteugvolume:

respirations-

volume corrente: $l$

volumen: $l$ (se ogsd (vedere anche $V_{\mathrm{T}}$ ) $V_{\mathrm{T}}$

stromaufwärts

stroomopwaarts

a monte

total: $l$

ansmura

total

transpulmonar

tráquea

transrespiratorio

ranstorácico

urbulento

volumen corriente:

tecido

totós

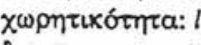

transmural, transparietal $\delta$ เ $\alpha \tau o เ \chi \omega \mu \alpha \tau \iota \kappa o \varsigma$

total

oגıKos

transpulmonar

traqueia

vevuoviKós

cpaxzía.

transrespiratorio

transtorácico

turbulento

SıаvarvevorıKós

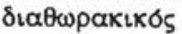

бтровucosins

avarvé $\mu \varepsilon v 0$ os

бүкоS: $l$

$\left(\beta \lambda \varepsilon \pi \varepsilon \varepsilon \pi\right.$ lons $\left.V_{T}\right)$

$\eta \pi \rho \circ \tau \alpha \varepsilon \pi \alpha v \omega$ por 


taliano

Español

Português

Eגイฑุvเxá

UNDW ultrasonically nebu- eau distillé nébulised distilled weter lise par utrasons

Ultraschall verne- ultrasoon vemeveld ultrasonisk for-

nebbia ultrasonica agua destilada tes Wasser gedestilleerd water stovetdestillere vand ebulizada mediante

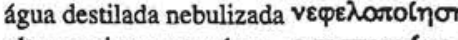

lised

mixed venous

gas volume: $l$

venös

veneus

vene, ven $\phi s$

venoso

venoso misto

venoso

Gasvolumen: $l$

gasvolume: $l$

luftumfang: $l$

volume di gas: $l$

venoso mezclado destilada inalada por $\mu \varepsilon$ vлєptixous nebulização ultrassónica

venoso

$\varphi \lambda \varepsilon \beta 1 x \propto \zeta$

venoso misturado,

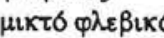

venoso misto

volume da gás;

oykos arplov: $l$

$V_{\mathrm{L}} \quad$ lung gas volume, airways: $l$ pulmonaire, y com- einschl. Gas in den incl. gas in de

luftumfang $\mathrm{i}$ vejenen: $l$ voies aériennes: $l$

$V_{\mathrm{T}} \quad$ tidal volume: $l$ volume courant: $l$ Atemzugsvolumen: ademteugvolume: see also (voyez aussi TV)

$V$ instantaneous gas débit gazeux instan- momentane Gas- momentane volumevolume flow: $1 \cdot \mathrm{s}^{-1}$ tané: $1 \cdot \mathrm{s}^{-1} \quad$ strömung: $l \cdot \mathrm{s}^{-1}$ stroom: $1 \cdot \mathrm{s}^{-1}$ strom): $l \cdot \mathrm{s}^{-1}$ maximal expiratory débit expiratoire flow: $l \cdot \mathrm{s}^{-1}$ (see also maximal: $l \cdot \mathrm{s}^{-1}$ maximaler exspimaximale expira- maksimal eksspiraMEF)

(voyez aussi MEF) $\quad l \cdot \mathrm{s}^{-1}($ siehe auch

$$
\text { MEF) }
$$

volumestroom: torisk volum

$V_{\max x} \quad$ MEF when $\mathrm{x} \%$ of débit expiratoire MEF wenn $\mathrm{x} \%$ der MEF wanneer nog $V_{\max }$ i det $\phi$ jeblik the FVC or TLC remains to be ex- $\mathrm{x} \%$ de la capacité noch auszuatmen haled: $1 \cdot \mathrm{s}^{-1}$ (see $\begin{array}{ll}x \% \text { de la capacité noch auszuatmen } & \\ \text { vitale expiratoire } & \text { sind: } l \cdot s^{-1}(\text { siehe }\end{array}$ forcée ou de la ca- auch $\mathrm{MEF}_{\mathrm{x} \text { r }}$ ) cité pulmonaire toreste à expirer: $l \cdot s^{-1}$

(voyez aussi MEF

difference in MEF différence de MEF Differenz in MEF Differenz in MEF between two condi- entre deux condi- zwischen zwei Be$\begin{array}{lll}\text { tions when } \mathrm{x} \% \text { of } & \text { tions lorsque } \mathrm{x} \% \text { de } & \text { dingungen wenn } \\ \text { the FVC or TLC } & \text { FVC ou TLC } & \mathrm{x} \% \text { der FVC oder }\end{array}$ (to be specified) (a spécifier) remains to be ex- reste à expirer: haled: $l \cdot s^{-1}($ see l. $\cdot \mathrm{s}^{-1}$ (voyez also $\triangle M E F$,

also volume aussi $\left.\left.\triangle \mathrm{MEF}_{\mathrm{xav}}\right) \quad \triangle \mathrm{MEF}_{\mathrm{xqv}}\right)$ TLC noch verbleiben: $l \cdot \mathrm{s}^{-1}$ GasvolumenBeschleunigung: $l \cdot \mathrm{s}^{-2}$ $\mathrm{x} \%$ van de FVC of $\mathrm{x} \%$ af FVC eller TLC moet worden TLC ikke er uitgeademd: $l \cdot \mathrm{s}^{-1}$ udåndet: $l \cdot \mathrm{s}^{-1}(\mathrm{se}$ (zie ook MEF

verschil in $V_{\text {max }}$ tus- forskel i MEF malt differenza di flusso en twee condities under to forskellige massimo in due an de FVC of TLC hvor $x \%$ of FVC (te specificeren) eller TLC endnu moet worden uitge- ikke er udåndet: ademd: $l \cdot \mathrm{s}^{-1}$ (zie $\quad l \cdot \mathrm{s}^{-1}$ ook $\triangle M E F$, ook $\triangle \mathrm{MEF}_{\mathrm{xsv}}$ ling: $1 \cdot \mathrm{s}^{-2}$ $l \cdot \mathrm{s}^{2}$ , inkluderer gas incluso il gas nelle gas, incluyendo el gas incluindo o das vias rumfanget $i$ luft- via aeree: n las vías aéreas: $l$ aéreas: $l$

respirationsvolumen volume corrente: $l$ volumen corriente: $l$ volume corrente: $l$

(tidalvolumen): l (vedere anche TV) (ver también TV) (ver também TV) (se ogsá TV) eblikkeligt gas- flusso istantaneo:

flujo instantáneo flow (volumen- $\quad l \cdot \mathrm{s}^{-1}$

$$
\text { (se ogsa MEF) }
$$
også MEF $\mathrm{MEv}_{\times \%}$ ) massimo: $1 \cdot \mathrm{s}^{-1}$ (vedere anche MEF) de gas: $l \cdot \mathrm{s}^{\cdot}$

débito gasoso :

flujo espiratorio máximo: $l \cdot \mathrm{s}^{-1}$

flusso massimo espi- $\mathrm{MEF}$ curndo $\mathrm{x} \%$ MEF quado fata ratorio a determinati de la FVC 0 TLC aún expirarar $x \%$ de FVC livelli di capacità debe ser espirado: $l \cdot \mathrm{s}^{-1}$ resta nos pulmões $\mathrm{x} \%$ vitale o di capacita (ver tambien MEF ) de TLC: $1 \cdot \mathrm{S}^{-1}$ (ver polmonare totale: $l \cdot \mathrm{s}^{-1}$ vedere anche $\mathrm{MEF}_{x, y /}$ ) tamberm MEF ) .

diferencia de $M$ MFF

Jiforença de $M$ CEF entre dos condiciones tuando el porcentaje eterminado em duas della CVF o della (debe especificarse) $x \%$ de FVC, ou resta CPT (da specificare): aún debe ser espirado: pulmōes $x \%$ de TLC $\mathrm{s}^{-1}$ er. $l \cdot s^{-1}$ (vedere anche $\quad l \cdot s^{-1}$ (ver también (a especificar): $l \cdot \mathrm{s}^{-}$

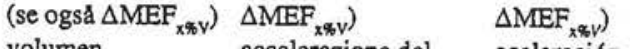
volumenaccelerazione de celeración de ver também $\triangle \mathrm{MEF}_{\mathrm{x}, \mathrm{p}}$

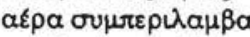

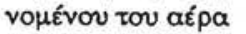
otous aepaywyous: $l$

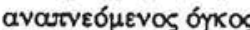
( $\beta \lambda \varepsilon \pi \varepsilon \varepsilon \pi l o n \varsigma$ TV)

orıүulala poń órxov aepiov: $l \cdot s^{-1}$

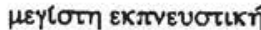
pơ: $1 \cdot \mathrm{s}^{-1}(\beta \lambda \varepsilon \pi \varepsilon \varepsilon$ exión (MEF)

MEF $6 \tau \alpha v \times \%$ זms

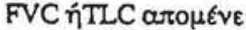

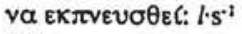
( $\beta \lambda \varepsilon \pi \varepsilon \varepsilon \pi$ ध一) $\mathrm{MEF}_{n \times p}$ )

Slayopá m̧s MEF

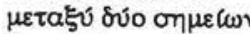
óav $x \%$ ms FVC r $\operatorname{TLC}(v \alpha \circ \rho\llcorner\sigma \theta \varepsilon C)$

a

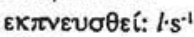
( $\beta \lambda \varepsilon \pi \varepsilon \varepsilon \pi l o n s$ $\triangle M E F_{x s v)}$ $\begin{array}{lll} & & \\ & & \end{array}$ 


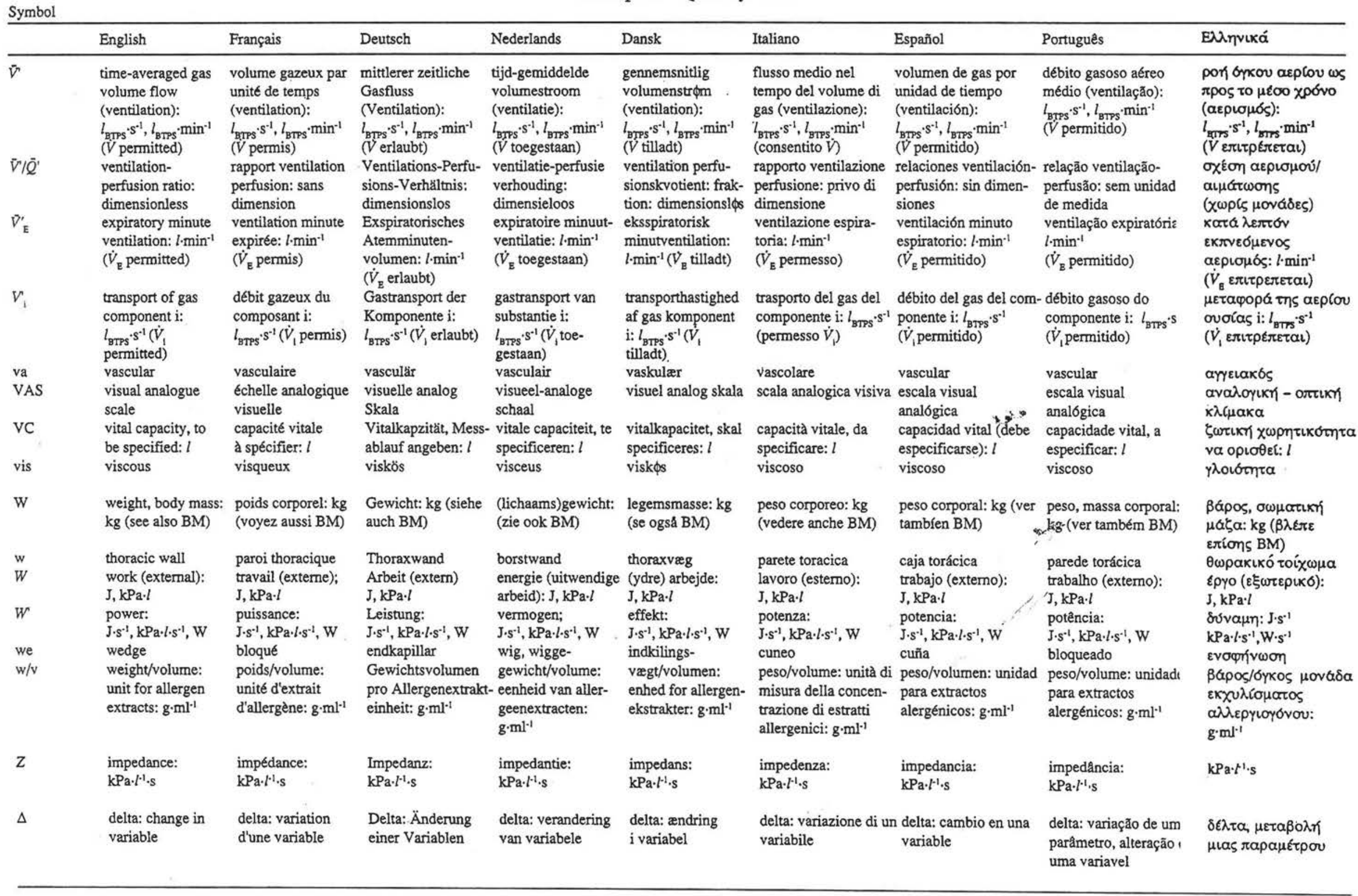


Italiano

Español

Portugues

EגไๆทเKá

$\sigma$

surace

Deutsch

Dansk

tensio

$\sigma$

surface

tension super-

$\begin{array}{lll}\text { Oberflächenspan- oppervlaktespan- } & \text { overfladesp } \\ \text { nung: } \mathrm{N} \cdot \mathrm{m}^{-1} & \text { ning: } \mathrm{N} \cdot \mathrm{m}^{-1} & \text { ding } \mathrm{N} \cdot \mathrm{m}^{-1}\end{array}$

$$
\mathrm{N} \cdot \mathrm{m}^{-1}
$$

$\mathrm{N} \cdot \mathrm{m}^{-1}$

$\mathrm{N} \cdot \mathrm{m}^{-1}$

eлLраvelaky ráo):

reaction rate coef-

coefficient de réac-

eaktionsgeschwin- reactieverhou

hrmoglobins reak- tasso di reazione

$\mathrm{N} \cdot \mathrm{m}^{-1}$

superficial:

globin for compo-

globine pour le

digkeits-Koeffizient coëfficiënt van

tionshastigheds-

r.

coeficiente de reaccazo

nent $\mathrm{i}$

compossant i:

Komponente i: stof i:

koefficient for kom- la componente i:

de la hemoglobina

da hemoglobina para

бurce

$\mathrm{mmol} \cdot \mathrm{min}$

Examples of notations

$P_{\mathrm{A}, 02} \quad$ alveolar oxyge

$P_{\mathrm{A}, 02} \quad$ alveolar oxygen

pression alvéolaire alveolärer Sauer$\mathrm{mmol} \cdot \mathrm{min}^{-1} \cdot \mathrm{kPa}^{-1}, l^{-1} \quad \mathrm{mmol} \cdot \mathrm{min}^{-1} \cdot \mathrm{kPa}^{-1} \cdot l^{2}$

componente i:

ons ms aur

vडs $\mu \varepsilon$ mv ougra d'oxygène

alveolaire (partiele)

$P_{\text {oes }} \quad$ oesophageal

pressure

pression

oesophagienne

$P_{\text {Ltivit }}$ frictional pressure pression friction-

in the lung tissue

nelle du tissu

Oesophagusdruck

zuurstofdruk

alveor

pressione parziale

presión alveolar de

$\mathrm{mmol} \cdot \mathrm{min}^{-1} \cdot \mathrm{kPa}^{-1} \cdot l^{-1}$

min

$P_{(A-x), 002 \quad \text { alveolar-arterial }}$

pulmonaire

visköser Lungen-

gewebedruck

visceuze druk in

oesofagustryk

di ossigeno alveolare oxígeno

pressão parcial

alveolar do oxigénio

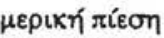

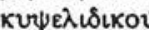

oร̌uyơvou

alveolar-arterial

ence de

alveolär-arterielle alveolair-arterieel

friktionsbetinget

pressione esofagea presión esofágica

pressåo esofágica

оюоффүчкт $\pi \varepsilon \varepsilon \sigma \eta$

for $\mathrm{CO}_{2}$

capillaire pour $\mathrm{CO}_{2}$

$C_{\text {L,dyn }} \quad$ dynamic

compliance of the

compliance

kymet

pressione conseguente presión de fricción pressão friccional do

alle resistenze viscose en el téjido pulmonar tecido pulmonar

del tessuto polmonare

alveolo-arteriel

differenza alveolo-

diferencia alveolo- diferença alveolo-

tryk-differens for capillare di pressione arterial de presion $\gg$ a arterial de $\mathrm{CO}_{2}$

lung

pulmonaire

$\mathrm{CO}_{2} \quad \mathrm{CO}_{2}$

parziale di $\mathrm{CO}_{2}$ parcial de $\mathrm{CO}$

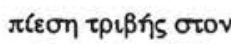

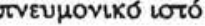

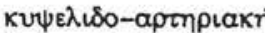

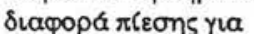

to $\mathrm{CO}_{2}$

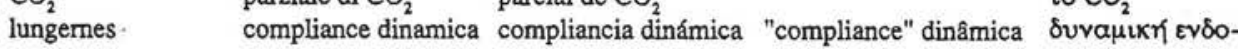

Lungencompliance compliantie van dynamiske

del polmone

del pulmón

do pulmåo

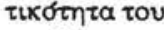

changement de Bronchialdruck- bronchiale druk- compliance

bronchial pressure pression bronchique schwankung verandering bronkietryk

or bronchial pressure

variazione della

cambio de presión

variação da pressão

$\pi v E v \mu O v \alpha$

pressione bronchiale bronquial

brêtätica

blood flow

shunt sangui

anatomischen Shunt bloedstroom

anatomisk gennem

flusso ematico dello

cortocircuito

"shunt" anatómico

$\mu \varepsilon \tau \alpha \beta 0 \lambda . \gamma$ mS

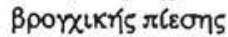

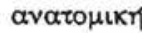

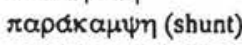

tns auratiktis poris 